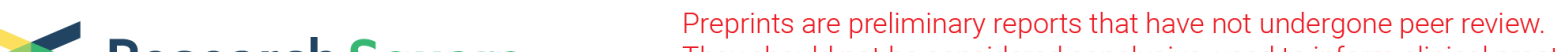 Research Square They should not be considered conclusive, used to inform clinical practice, or referenced by the media as validated information.
}

\section{Lipidomics Profiling of Goose Granulosa Cells \\ Model of SCD Function Identifies a Pattern of Lipid Droplets Associated with Follicle Development}

Xin Yuan ( $\square$ nihaoyuanxin88@outlook.com )

Sichuan Agricultural University - Chengdu Campus https://orcid.org/0000-0002-7130-5990

Shenqiang $\mathrm{Hu}$

Sichuan Agricultural University - Chengdu Campus

Liang Li

Sichuan Agricultural University - Chengdu Campus

Chunchun Han

Sichuan Agricultural University - Chengdu Campus

Hehe Liu

Sichuan Agricultural University - Chengdu Campus

Hua He

Sichuan Agricultural University - Chengdu Campus

Lu Xia

Sichuan Agricultural University - Chengdu Campus

Jiwei Hu

Sichuan Agricultural University - Chengdu Campus

Bo Hu

Sichuan Agricultural University - Chengdu Campus

\section{Mingxia Ran}

Sichuan Agricultural University - Chengdu Campus

Yali Liu

Sichuan Agricultural University - Chengdu Campus

Jiwen Wang

Sichuan Agricultural University - Chengdu Campus

\section{Research}

Keywords: SCD, gene expression, goose granulosa cells, ovarian follicles, lipid droplets

Posted Date: March 12th, 2021

DOl: https://doi.org/10.21203/rs.3.rs-281728/v1 
License: (c) (i) This work is licensed under a Creative Commons Attribution 4.0 International License. Read Full License 


\section{Abstract}

Despite their important functions and nearly ubiquitous presence in cells, an understanding of the biology of intracellular lipid droplets (LDs) in goose follicle development remains limited. An integrated study of lipidomic and transcriptomic analyses was performed in a cellular model of stearoyl-CoA desaturase (SCD) function, to determine the effects of intracellular LDs on follicle development in geese. Numerous internalized LDs, which were generally spherical in shape, were dispersed throughout the cytoplasm of granulosa cells (GCs) by confocal microscopy analysis, with altered SCD expression affecting LD content. GCs lipidomics profiling showed that the majority of the differentially abundant lipids classes were glycerophospholipids, including PA, PC, PE, PG, PI and PS, and glycerolipids, including DG and TG, which enriched the glycerophospholipid metabolism, sphingolipid metabolism and glycerolipid metabolism. As compared with lipidomics, transcriptomics provided differentially expressed genes (DEGs), part of which were assigned to lipid-related Gene Ontology slim terms. More DEGs were assigned in the SCD-knockdown group than in the SCD-overexpressed group. Integration of the differentially significant genes and lipids into pathway enrichment analysis identified potentially targetable pathways in glycerolipid/glycerophospholipid metabolism. These results demonstrated the importance of lipids in providing novel insights to understand follicle development, thus providing a potential lead to new avenues for deciphering the underlying mechanisms of lipid-mediated follicle development.

\section{Introduction}

Lipids are a highly diverse class of molecules key to many cellular processes, including energy storage, structural moieties, signaling molecules, and protein modification (Santos and Preta, 2018; Storck et al., 2018). Lipids have been loosely defined as biological substances that are generally hydrophobic in nature and, in many cases, soluble in organic solvents. These chemical properties include a broad range of molecules, including fatty acids, phospholipids, sterols, sphingolipids, terpenes, and others, with each having a role in living systems (Brown and Marnett, 2011). Lipid metabolism involves numerous interwoven pathways; these networks are essential for regulating cell function, which is influenced by many external factors. Imbalanced changes in lipid composition in this system may even affect various physiological functions of the cell (Bochkov et al., 2010). Lipid overload results in lipotoxicity that initially manifests as organelle dysfunction, ultimately leading to cell death and tissue damage (Feldstein et al., 2004; Ozcan et al., 2006). Evidently, deregulation of this network contributes to the onset of pathology as lipids are tightly regulated, spatially and temporally, in various parts of the organism (Liu and Xun, 2013; Goldberg et al., 2018). A number of lipid molecular species have been identified as potential biomarkers involved in several diseases, including, epidemic diseases, cancers, inflammations, and genetic diseases (Lydic et al., 2018).

Intracellular lipid droplets (LDs) occur through the deposition of lipid complex molecular pathways. They are found in a wide variety of cell types, and are viewed as highly dynamic organelles with unique spherical structures, whose primary neutral lipids are mainly sterol esters and triacylglycerols (TGs) (Den Brok et al., 2018; Olzmann and Carvalho, 2019). LDs affect cell and organ health and contribute to the 
dynamics of biological membranes, energy storage and expenditure, and to conveying messages at all cellular levels, including the nucleus (Onal et al., 2017; Mike et al., 2019). Despite their important functions and nearly ubiquitous presence in cells, many aspects of LD biology are unknown. We have previously confirmed that de novo lipogenesis occurs in goose GCs (Wen et al., 2019) and that LD accumulation capacity depends on the stage of GCs during goose follicle development (Gao et al., 2019). It has been reported that lipid metabolism in bovine, sheep, and human GCs is pivotal for oocyte maturation, fertilization, and early embryonic development (Mcevoy et al., 2000; Campbell et al., 2010; Hu and Qiao, 2011; Elis et al., 2015). Lipid content in follicles differs among species, as reported in studies performed in porcine (Campagna et al., 2010), bovine (Abe et al., 2002), and canine oocytes (De Lesegno et al., 2008), wherein LD number, size, and distribution were analyzed. However, an understanding of the biology of LDs in GCs remains limited. Recently, the role of stearoyl-CoA desaturase (SCD) as a regulator of fatty acid desaturation in goose GCs was identified, whereby metabolite changes in lipid-related metabolism, was found to be closely related to follicular development (Yuan et al., 2020). Although this observation indicated that such links might exist, it is unclear whether LDs play a role in goose follicle development, and which lipid species or classes might be affected. It is therefore hypothesized that lipidomic analysis of the goose GCs at the level of individual lipid molecular species might help to elucidate such a mechanism.

Lipidomics is a comprehensive characterization of an organism's differences in individual lipid molecular species and their biological roles with respect to the expression of proteins involved in lipid metabolism and function (Liu, 2017; Lange et al., 2018). Thus, to obtain a detailed view of lipid metabolism, it is crucial to analyze the full lipid profiles of the individual lipids by using a lipidomics approach. This is the first time that lipidomics combined with transcriptomics analysis has been applied in the GC model of SCD function to identify alterations in the specific gene regulation, lipid classes, and lipid-mediated signaling processes that are involved in LDs associated with follicle development in geese. The detailed characterization of lipid classes and species provided in this study may be useful to understand the mechanism of intramyocellular LDs, and analysis by integrating two-omics datasets will broaden our understanding of lipid alterations in lipid-gene networks in GCs associated with follicle development.

\section{Materials And Methods}

\subsection{Animals and sample collection}

Geese (from a maternal line of Tianfu goose) were raised under natural temperature and light conditions at the experimental station of waterfowl breeding at Sichuan Agricultural University. Six geese with regular laying sequences were randomly selected as experimental samples and sacrificed by cervical bleeding under anesthesia, $2 \mathrm{~h}$ after oviposition. A pool of ovarian follicles was immediately collected, and the outer connective tissue removed. The granulosa layers were isolated as described previously (Gilbert et al., 1977). 


\subsection{Cell culture and siRNA transfection}

The granulosa layer was dispersed by incubation in $0.1 \%$ type II collagenase (Sigma, USA) in Dulbecco's modified Eagle's medium (DMEM, HyClone, USA) for $10 \mathrm{~min}$ in a $37{ }^{\circ} \mathrm{C}$ water bath. After incubation, cells were dispersed with a pipette and pelleted by centrifugation at $1,000 \times \mathrm{g}$ for $10 \mathrm{~min}\left(20^{\circ} \mathrm{C}\right)$. The supernatant was discarded, and the cells resuspended in $3 \mathrm{ml}$ of fresh basic medium without collagenase and centrifuged. The washing procedure was repeated twice. The GCs were dispersed in DMEM supplemented with $1 \%$ antibiotic/antimycotic solution (Solarbio, China) and $3 \%$ fetal bovine serum (Gibco, USA). Transient and stable transfections to the GC cellular model of SCD function (specific SCD overexpress and knockdown) were performed using Lipofectamine ${ }^{\circledR} 3000$ and Lipofectamine ${ }^{\text {TM }}$ RNAiMAX Transfection Reagent (Invitrogen Co.), according to manufacturer's protocol. SCD siRNA-210 and siRNA405 were used to achieve SCD mRNA knockdown, with scrambled siRNA as a negative control. The extent of SCD mRNA knockdown was measured as a percentage compared with the scrambled SiRNA. SCDspecific overexpression was used to achieve SCD mRNA overexpression, termed pEGFP-N1/SCD, pEGFP$\mathrm{N} 1 /$ empty as negative control, and the other control with no transfection components. The extent of SCD mRNA overexpression was measured as a percentage compared with the pEGFP-N1/empty and no transfection components. Transfection efficiency was determined as previously described (Yuan et al., 2020).

\subsection{Oil Red 0 staining}

To determine LDs accumulation of GCs after transfection, the cells were stained with Oil Red 0 solution (Sigma Chemical Co., St. Louis, MO, USA). After $48 \mathrm{~h}$ of transfection, the cultured GCs were washed with phosphate-buffered saline (PBS) and fixed with 10\% formaldehyde at room temperature. The cells were stained with $0.5 \mu \mathrm{g} / \mathrm{mL}$ Oil Red $O$ solution and photographed using an optical microscope system (Nikon ECLIPSE 90i) at $200 \times$ magnification. The LDs were dissolved in isopropanol and absorbance was measured at a wavelength of $540 \mathrm{~nm}$ by using a microplate reader (Thermo Varioskan, USA). The relative lipid content was calculated using the following equation: Relative LD content $(\%)=$ Sample OD $/ \mathrm{mg}$. $\mathrm{mL}^{-1}$ protein.

\subsection{Immunofluorescence staining and confocal microscopy}

For photographic documentation of LD localization in goose GCs, live cells were stained with 4,4-difluoro3a,4a diaza-s-indacene (Bodipy 500/510, Thermo Fisher), wheat germ agglutinin (WGA, Alexa Fluor 594 conjugate, Invitrogen), and Hoechst dyes (33258, Invitrogen) for labeling the LDs, membrane, and nucleus, respectively. Briefly, cells on culture plates were rinsed with PBS and stained for 5 min with 2 $\mu \mathrm{g} / \mathrm{ml}$ Bodipy 500/510. After staining, cells were washed three times with PBS for $5 \mathrm{~min}$. The cells were then stained for $5 \mathrm{~min}$ with $5 \mu \mathrm{g} / \mathrm{ml} \mathrm{WGA}$ and $5 \mu \mathrm{g} / \mathrm{ml}$ Hoechst 33342. After staining and incubation, the cells were washed thrice with PBS for 5 min. Finally, cells were fixed in formaldehyde for $10 \mathrm{~min}$. 
Confocal fluorescence scanning microscopy images of fixed cells were collected with a confocal laser scanning microscope (FV1200, Olympus) mounted on an IX83 inverted microscope (Olympus). For treblecolor images, the $550 \mathrm{~nm}$ laser line was used to image cells stained with Bodipy 500/510, the $635 \mathrm{~nm}$ laser line was used to image cells stained with WGA and the $543 \mathrm{~nm}$ laser line was used to image cells stained with Hoechst 33258.

\subsection{Sample preparation and extraction}

For lipidomic analysis, each group of cells, SCD-overexpression group (referred to as LS); the GFP vector group (referred to as LG); the control group (referred to as LN); two independent siRNA groups (siRNA-210 and siRNA-470, referred to as $L T$ and LF, respectively); and a scrambled siRNA group (referred to as $L C$ ), were placed in liquid nitrogen for $2 \mathrm{~min}$, then thawed on ice for $5 \mathrm{~min}$ and subjected to vortex blending. The cells were centrifuged at $12,000 \mathrm{rpm}$ at $4{ }^{\circ} \mathrm{C}$ for $10 \mathrm{~min}$. The supernatants $(300 \mu \mathrm{L})$ were homogenized with a $1 \mathrm{~mL}$ mixture containing methanol, MTBE, and an internal standard. This mixture was stirred for $2 \mathrm{~min}$, followed by addition of $500 \mu \mathrm{L}$ water, stirring for $1 \mathrm{~min}$, and centrifugation at 12,000 rpm at $4{ }^{\circ} \mathrm{C}$ for $10 \mathrm{~min}$. A total of $500 \mu \mathrm{L}$ of the supernatant obtained was extracted and concentrated. The powder was dissolved with $100 \mu \mathrm{L}$ mobile phase B (0.1\% acetic acid), and the supernatant $(200 \mu \mathrm{L})$ was transferred to an LC-MS sampling vial with an inner liner, for LC-MS analysis. Quality control (QC) samples were produced by pooling equal aliquots taken from each individual sample in the analytical run together. These QC samples were used to monitor the stability and reproducibility of the analytes in the samples, during the analysis.

For RNA-sequencing analysis, each group of cells was extracted from three independent biological replicates by using TRIzol reagent (Invitrogen, USA) according to the manufacturer's instructions. RNA quality was confirmed on $1 \%$ agarose gels. RNA purity and concentration were determined using a NanoPhotometer ${ }^{\circledR}$ spectrophotometer (IMPLEN, CA, USA) and Qubit ${ }^{\circledR}$ RNA Assay Kit (Qubit $\circledast 2.0$ Fluorometer; Life Technologies, CA, USA) according to the manufacturer's instructions. RNA integrity was assessed using the RNA 6000 Nano Assay Kit (Bioanalyzer 2100 system; Agilent Technologies, CA, USA).

\subsection{Lipidomics profiling}

Lipidomics profiling was performed by MetWare (Wuhan, China) using an LC-ESI-MS/MS system (UPLC, Shim-pack UFLC SHIMADZU CBM A system, https://www.shimadzu.com/; MS, QTRAP® System, https://sciex.com/). LIT and triple quadrupole (QQQ) scans were acquired on a triple quadrupole-linear ion trap mass spectrometer (QTRAP), QTRAP® LC-MS/MS System, equipped with an ESI Turbo lon-Spray interface, operating in positive and negative ion mode, and controlled by Analyst 1.6.3 software (Sciex). Qualitative analysis of primary and secondary MS data was carried out by comparison of the accurate precursor ions (Q1), product ions (Q3) values, retention time (RT), and fragmentation patterns with those obtained by injecting standards at the same conditions if the standards were available (Sigma-Aldrich, USA), or was conducted using a self-compiled database MWDB (MetWare, China). The quantitative 
analysis of metabolites was based on the MRM mode. The characteristic ions of each metabolite were screened using a QQQ mass spectrometer to obtain the signal strengths. Integration and correction of chromatographic peaks was performed using Progenesis QI software (Waters Co., Milford, MA, USA). The corresponding relative metabolite contents were represented as chromatographic peak area integrals. In addition, accurate masses of features representing significant differences were searched against the METLIN, Kyoto Encyclopedia of Genes and Genomes (KEGG), HMDB, and LIPIDMAPS databases.

\subsection{RNA-Sequencing}

Sequencing libraries were performed by Novogene (Beijing, China) and generated using the NEBNext® Ultra ${ }^{\text {TM }}$ RNA Library Prep Kit (Illumina ${ }^{\circledR}, N E B$, USA) following the manufacturer's instructions. Clustering of the index-coded samples was performed on a cBot Cluster Generation System by using the TruSeq PE Cluster Kit v3-cBot-HS (Illumina) according to the manufacturer's instructions. After cluster generation, the library preparations were sequenced on an Illumina HiSeq (Illumina, USA) platform and 125 bp/150 bp paired-end reads were generated.

\subsection{Bioinformatic and statistical analyses of data}

Raw LC-MS data was filtered, identified, integrated, corrected, aligned, and normalized using Progenesis QI software (Waters Co., Milford, MA, USA). A data matrix of RT, mass-to-charge ratio, and peak intensity was obtained. The processed data was analyzed using principal component analysis and orthogonal correction partial least squares discriminant analysis (PC) using SIMCA-P14.0 software (Umetrics, Umeå, Sweden). Differentially abundant metabolites between dietary treatments were identified from variable importance in projection (VIP) from OPLS-DA and Student's t tests (VIP $>1$ and $\mathrm{P}<0.05)$. Metabolites were identified from public databases, including MassBank (http://www.massbank.jp/), KNApSAcK (http://kanaya.naist.jp/KNApSAcK/), Human Metabolome Database (http://www.hmdb.ca/), and METLIN (https://metlin.scripps.edu/). The KEGG database (http://www.genome.jp/kegg/) was used to view the enriched pathways of the different metabolites. Hierarchical clustering analysis and heat map analysis were conducted using the $\mathrm{R}$ package, version 3.3.1. The raw read counts were normalized considering both the different depths of sequencing among the samples and the gene GC content. Normalization was performed using the EDASeq package. We considered all differentially expressed genes (DEGs) with a $P$ value $₫ 0.05$ after false discovery rate correction. The putative functions of DEGs were investigated by Gene Ontology (GO) enrichment analysis using the GOseq R package (Young et al., 2010), in which gene length bias was corrected. $G 0$ terms with a corrected $P$ value $<0.05$ were considered significantly enriched. KOBAS software was used to test the statistical enrichment of DEGs in KEGG pathways (Mao et al., 2005). Statistical plots were calculated by using Origin version 6.1.

\section{Results}




\subsection{LDs distributions in goose GCs}

Previous studies have demonstrated that de novo lipogenesis exists in goose ovarian GCs and has lipid accumulation capacity (Gao et al., 2019; Wen et al., 2019). To investigate the localization of LDs in goose GCs, confocal microscopy was used to analyze LD location in goose GCs stained by BODIPY (493/503, green). GC cells contained numerous internalized LDs that were generally spherical in shape and dispersed throughout the cytoplasm (Figure 1). The dispersed LDs were clumped to form clusters. The dispersed LDs mobility was from the perinuclear regions to the extracellular domain.

\subsection{Altered SCD expression affected LD content}

To further investigate the effect of SCD expression on LDs in GCs, the LDs were stained with Oil Red $O$ after SCD-overexpression and knockdown culturing (Figure 2A and B). The accumulation of LD content was quantified using equations (see Materials and methods section for details), compared with the control group, overexpression of SCD significantly stimulated the accumulation of LD content. However, SCD knockdown significantly inhibited the accumulation of LDs

\subsection{Analysis of lipid classes}

A total of 662 species of lipids were identified in the GC samples by LC-MS/MS analysis (Supplementary Figure 1). There were 183 different lipids identified in the LN vs. LS comparison (165 upregulated and 18 downregulated) based on fold-change analysis (VIP >1, fold-change (FC) > 1.2), 85 lipids in the LG vs. LS comparison (36 upregulated and 49 downregulated), 90 lipids in the LC vs. LT comparison (45 upregulated and 45 downregulated), and 106 lipids in the LC vs. LF comparison (86 upregulated and 20 downregulated). Glycerophospholipids and glycerolipids were the predominant lipid species (Figure 3 and Supplementary Table 1). Venn diagram analysis revealed 26 overlapping lipids in the SCD-overexpression group, 35 overlapping lipids in the SCD-knockdown group, and 9 overlapping lipids in the SCDoverexpression and SCD-knockdown groups (Supplementary Figure 2). As shown in Table 1 and Figure 4, 19 lipids followed the same trend, while 7 lipids showed the opposite pattern as compared to the two overexpressed group (LN vs. LS comparison and LG vs. LS comparison). 33 lipids followed the same trend, while 2 lipids showed the opposite pattern as compared to the two knockdown group (LC vs. LT comparison and LC vs. LF comparison). 7 lipids followed the same trend, while 2 lipids showed the opposite pattern between the overexpressed and knockdown groups.

\subsection{Cross comparisons to and within each group}

With the aid of the KEGG database, metabolic pathways were summarized in Supplementary Table 2 based on the identified lipids. A total of 183 of these differentially expressed lipids were associated with 57 metabolic pathways in the LN vs. LS comparison, 85 lipids with 40 metabolic pathways in LG vs. LS 
comparison, 90 lipids with 46 metabolic pathways in LC vs. LT comparison, and 106 lipids with 42 metabolic pathways in LC vs. LF comparison. Figures $3 \mathrm{~A}$ and B represent the perturbed SCD pathways. For $L N$ versus $L S$, metabolism changed for glycerophospholipid, arachidonic, sphingolipid, and glycerolipid; for LG versus LS, glycerophospholipid, sphingolipid, glycerolipid, and inositol phosphate; for LC versus LT, glycerophospholipid, sphingolipid, glycerolipid, and inositol phosphate; and for LC versus LF, glycerophospholipid, sphingolipid, glycerolipid, and inositol phosphate.

\subsection{RNA-seq investigation of overexpression and knockdown of SCD expression in GCs}

To obtain a comprehensive view of the role of the SCD gene in GCs, the six groups of cells (OS, OG, and NC; ST, SC, and SF) were subjected to RNA-seq analysis. According to the RNA-seq data, 51.56, 53.78, $55.70,57.70,51.65$, and 56.19 million clean reads were obtained from the NC, OS, OG, SC, ST, and SF samples, respectively. For each sample, $37.69,38.67,40.04,42.60,37.88$, and 41.30 million unique reads could be mapped to the current version of the goose genome (Anser cygnoides) (Table 2). The basic characteristics of the tags in each sample library are shown in Supplementary Table 3.

\subsection{Identification and functional enrichment of DEGs}

A total of 679 DEGs were identified in the GCs after SCD-overexpression and knockdown (Supplementary Figure 3), including 125 DEGs (41 upregulated and 84 downregulated) in NC vs. OS comparison, 121 DEGs (66 up- and 55 downregulated) in OG vs. OG comparison, 199 DEGs (57 upregulated and 142 downregulated) in SC vs. ST comparison, and 306 DEGs (92 upregulated and 214 downregulated) in SC vs. SF comparison (Figure 4A and B).

To characterize differences in lipid metabolism between the transcriptomes of the SCD-overexpression and SCD-knockdown groups, we used the GO slim terms of the complex Gene Ontology annotations. The results showed that DEGs were assigned to 20 lipid-related GO terms in NC vs. OS comparison, 21 lipidrelated GO terms in OG vs. OS comparison, 63 lipid-related GO terms in SC vs. ST comparison, and 63 lipid-related GO terms in SC vs. SF comparison (Figure 7 and Supplementary Table 4). The top 20 pathways of KEGG functional analysis showed that SCD-overexpression had a significant effect on one carbon pool by folate, glycosphingolipid biosynthesis, ferroptosis, and influenza A; knockdown of SCD had a significant effect on sphingolipid metabolism, proteasome, mTOR signaling pathway, hedgehog signaling pathway, glycosphingolipid biosynthesis (metabolism), ether lipid metabolism, amino sugar and nucleotide sugar metabolism, and protein processing in the endoplasmic reticulum (supplementary Figure $4 A$ and $B$ ). 


\subsection{The level of coordination between transcript and lipidomic data}

Monitored responses in parallel on the transcriptome and the lipid level were carried out, thus allowing one to compare the level of coordination between both molecular readouts. To perform this analysis, a holistic co-clustering approach is shown in Figure 8. Perturbed SCD in GCs influenced mRNA and metabolite metabolism in glycerolipid/glycerophospholipid metabolism, glycine, serine, and threonine metabolism, and sphingolipid metabolism, which are associated with lipid-related metabolism pathways. However, SCD knockdown in GCs influenced more complicated lipid-related metabolism pathways, including fatty acid degradation/elongation, inositol phosphate metabolism, phosphatidylinositol signaling system, alpha-linolenic acid metabolism, arachidonic acid metabolism, and linoleic acid metabolism. Additionally, a targeted approach using prior biological knowledge in conjunction with canonical correlation analysis (CCA) revealed that lipids from glycerolipid/glycerophospholipid metabolism were subjected to a CCA together with transcript data of all those pathways as derived from SCD knockdown in GCs (Figure 9). We are also interested in other lipid-related pathways subjected to a CCA together with transcript data (Supplementary Figure 4). However, no similar CCA results were observed in SCD-overexpression in GCs.

\section{Discussion}

In the present study, we used a GC model of SCD function and performed a detailed study on cellular lipid metabolism using lipidomic profiling in order to evaluate the lipid molecular changes in a detailed manner. The method is based on ultra-high performance liquid chromatography combined with quadrupole-time-of-flight mass spectrometry and gas chromatography mass spectrometry, which allow for the analysis of hundreds of molecular lipids from a single sample run (Chen et al., 2013). Over 660 lipids were identified, including phospholipids, ceramides, diacylglycerol and triacylglycerols, cholesterol esters, and fatty acid compositions (Fig. 3). Among these lipids, glycerophospholipids and glycerolipids were the predominant species. Additionally, altered SCD expression had the greatest impact on glycerophospholipid metabolism, this lipid-related pathway contains the most key lipid, with over 6 representative metabolites: PC (16:0/20:3); PC (18:1/18:4); PC (18:2/20:3); LPC (18:3/0:0); PE (22:2/12:0); and PS (20:4/20:0). FFA (22:2) and TG (14:0/22:0/22:2) were glycerolipid metabolism representative metabolites, and sphingolipid metabolism (Figs. 4 and 5).

Large diversity exists in the structures and functions of the vast array of lipid species, with intracellular levels of lipids tightly regulated by a network of metabolic pathways to sustain normal cellular functions (Tang, 2016). Glycerophospholipids make up phospholipids, which play both structural and metabolic roles in cells. Therefore, the level and species of phospholipids may reflect in the biochemical and physiological conditions of cells (Dowhan, 1997). Phospholipids are important for determining LD formation and are attributed to the formation of large LDs fusion (Han et al., 2020). Glycerolipid metabolism is altered by fatty acids and, it induces LD formation (Pathmaperuma et al., 2010). Blocking 
PC synthesis caused elevated SREBP-1-dependent gene transcription and LD accumulation in mouse liver and human cells, and the ratio of PE to PC in the LD surface tended to affect LD number and size (Walker et al., 2011; Listenberger et al., 2018). The present study provided the lipid species composition and lipid metabolism of the GC model of SCD function. Given the important biological activities of many lipids, such information could provide potential insights into the mechanisms of internalized lipid-mediated follicle development.

We further investigated whether altered SCD expression of GCs affects gene expression involved in lipid metabolism. We observed that altered SCD expression resulted in many DEGs assigned to lipid-related GO terms involved in lipid binding, lipid transport, lipid localization, LD and so on. Compared with the SCD-overexpression group, more DEGs were identified and assigned to lipid-related GO terms in the SCDknockdown group (Fig. 7 and Supplementary Table 4). These results indicated that SCD regulates the lipid metabolic processes during goose follicle development. Particularly, SCD-knockdown may mobilize more genes associated with lipid metabolism. Combined analysis of lipid and gene expression profiles showed that more complicated lipid-related metabolism pathways were significantly clustered, especially in the SCD-knockdown group.

Numerous internalized LDs were dispersed throughout the cytoplasm of GC (Figs. 1 and 2). However, in steroidogenic tissues such as the ovary, the ovarian follicles of almost all mammals contained LDs, which were prominently and generally non-pathogenic, except in cases of genetic disorders of steroidogenesis (Paulini et al., 2014). We speculate that there is a relationship between LDs and energy supply or the storage of cholesteryl esters during goose follicle development. Cholesterol esters were significantly upregulated in SCD overexpress and knockdown groups, particularly CE (20:4) (Fig. 4C). Previous results demonstrated intracellular metabolite changes where cholesterol was mostly upregulated in the SCD-overexpressed group, whereas androsterone was upregulated in the SCDknockdown group (Yuan et al., 2020).

LDs make dynamic contacts with nearly every other organelle. Some contact activity may exist among the ER, endosomes, mitochondria, and peroxisomes in the cell. These contacts are thought to mediate the transfer of lipids (including phospholipids, sterols, and fatty acids) between the different cellular compartments (Gallardo-Montejano et al., 2016; Gao et al., 2017). Pharmacological inhibition of the enzymatic activity of fatty acid synthase significantly reduced the production of progesterone in bovine GCs, suggesting that ovarian steroidogenesis relies on lipid metabolism in GCs (Elis et al., 2015; Yenuganti et al., 2016). Additionally, LD accumulation capacity of goose GCs depends on the different stages of follicle development; with the stage of most accumulation capacity being F1 GCs, followed by F4-F2 GCs and pre-hierarchical GCs(Gao et al., 2019). As ovarian follicles grow, with changes in both the size and number of LDs in bovine (Dadarwal et al., 2015), the total intracellular LD content increases gradually with follicular growth. This exponential increase was demonstrated in large antral follicles in rats and goats (Sangha and Guraya, 1989; Sharma et al., 1996), with increase in LD numbers and size in GCs within a day after ovulation in rabbits (GURAYA and S., 1968). These results confirm that LDs are considered a source of energy for oocyte maturation (Sturmey et al., 2006; Silva et al., 2011). 
SCD catalyzes the rate-limiting step in the production of monounsaturated fatty acids (MUFAs), which are major substrates for the synthesis of triglycerides, wax esters, cholesterol esters, and phospholipids [56]. Recent research has revealed that alterations in SCD expression change the fatty acid profile of these lipids and have a significant role in lipid metabolism (Khor et al., 2013). The integrated optical density of the LDs is related to the amounts of triglycerides in the LDs. Our results showed that altered SCD expression affected LDs in GCs. Overexpression of SCD stimulated the accumulation of LD content, whereas SCD knockdown inhibited the accumulation of LD content (Fig. 2C). Previous research has demonstrated that SCD is an important control point in lipid homeostasis in goose GCs (Yuan et al., 2020). Similar results suggest that increased hepatic SCD activity accelerates the last stage of TG synthesis and stimulates lipid accumulation (Matthew et al., 2008). These results provide direct evidence that SCD expression plays a major role in the triacylglycerol and phospholipid secretion process. Much evidence indicates that the direct anti-steatotic effect of SCD deficiency stems from increased fatty acid oxidation, reduced lipid synthesis (Dobrzyn et al., 2005) and inhibition of SCD decreases proliferation and apoptosis of cells (Iwai et al., 2016). Additionally, a link between SCD activity and LD size was previously observed in the nematode C. elegans (Xun et al., 2013), and cell lines cultured from patients with Berardinelli-Seip congenital lipodystrophy (BSCL) (Boutet et al., 2009) demonstrated that SCD activity is necessary for large-sized LDs. Recent studies have indicated that the size of LDs may influence the balance of lipogenesis and lipolysis in adipocytes and hepatic cells (Welte and Gould, 2017), with decreased SCD expression resulting in reduced accumulation of oleic and palmitoleic acids and consequently, fat cells with small LDs (Choi et al., 2000). However, the functional implications of the diverse sizes of LDs in goose GCs are poorly understood.

In summary, these results provide new evidence that altered SCD expression changed GC lipid profiles, genes, and regulated relevant metabolic pathways, which may implicate SCD as one of the major genes in the regulation of lipid secretion of goose GCs.

\section{Declarations}

\section{Ethics approval and consent to participate}

The animal experiments were reviewed and approved by the Committee of the School of Farm Animal Genetic Resources Exploration and Innovation Key Laboratory, College of Animal Science and Technology, Sichuan Agricultural University, China (permit no. DKY20170913). The experiments were performed in accordance with the Regulations for the Administration of Affairs Concerning Experimental Animals (China 1988). This study was conducted in accordance with the requirements of the Beijing Animal Welfare Committee. All efforts were made to minimize animal suffering.

\section{Consent for publication}

All the co-authors agreed to publish the final version of the present manuscript.

\section{Availability of data and materials}


The authors acknowledge that the data presented in this study must be deposited and made publicly available in an acceptable repository, prior to publication. And $\nabla$ the datasets generated and analyzed during the current study are available from the corresponding author on reasonable request.

\section{Competing interests}

The authors declare that the research was conducted in the absence of any commercial or financial relationships that could be construed as a potential conflict of interest.

\section{Funding}

This study was supported by a grant from the National Natural Science Foundation of China (No. 31672424 and 035Z2425), the China Agricultural Research System (No. CARS-42-4), the Open Fund of Farm Animal Genetic Resources Exploration and Innovation Key Laboratory of Sichuan Province (SNDKKF-201802), the Project of National Science and Technology Plan for the Rural Development in China (No. 2015BAD03B06), and the Key Technology Support Program of Sichuan Province (No. 2016NYZ0044).

\section{Authors' contributions}

$\mathrm{XY}$ and JW conceptualized and designed this study, reviewed this manuscript, and supervised this study. $\mathrm{XY}, \mathrm{SH}, \mathrm{LL}, \mathrm{CH}$ and $\mathrm{HL}$ performed the main experiments and analyzed the data. $\mathrm{HH}, \mathrm{LX}, \mathrm{JH}, \mathrm{BH}, \mathrm{MR}$ and $Y L$ participated in experimental animal management, tissue sampling, and data collection and analysis. $\mathrm{XY}$ drafted this manuscript.

\section{Acknowledgements}

None.

\section{References}

1. Abe H, Yamashita S, Satoh T, Hoshi H. Accumulation of Cytoplasmic Lipid Droplets in Bovine Embryos and Cryotolerance of Embryos Developed in Different Culture Systems Using Serum-Free or Serum-Containing Media. Mol Reprod Dev. 2002;61:57-66. doi. 10.1002/mrd.1131.

2. Bochkov VN, Oskolkova OV, Birukov KG, Levonen AL, Binder CJ, Stöckl J. Generation and Biological Activities of Oxidized Phospholipids. Antioxid Redox Signal. 2010;12(8):1009-59. doi. 10.1089/ars.2009.2597.

3. Boutet E, Mourabit E, Prot H, Nemani M, Khallouf M, Colard E, O., et al. Seipin deficiency alters fatty acid Delta9 desaturation and lipid droplet formation in Berardinelli-Seip congenital lipodystrophy. Biochimie. 2009;91:796-803. doi. 10.1016/j.biochi.2009.01.011.

4. Brown HA, Marnett LJJCR. Introduction to Lipid Biochemistry, Metabolism, and Signaling. Chem Rev. 2011;111(10):5817-20. doi. 10.1021/cr200363s. 
5. Campagna C, Bailey JL, Sirard M-A, Ayotte P, Bailey JL. An environmentally-relevant mixture of organochlorines and its vehicle control, dimethylsulfoxide, induce ultrastructural alterations in porcine oocytes. Reprod Toxicol. 2010;73(1):83-91. doi. 10.1016/j.reprotox.2008.03.003.

6. Chen W, Gong L, Guo Z, Wang W, Zhang H, Liu X, et al. A Novel Integrated Method for Large-Scale Detection, Identification, and Quantification of Widely Targeted Metabolites: Application in the Study of Rice Metabolomics. Mol Plant. 2013;6(6):1769-80. doi. 10.1093/mp/sst080.

7. Choi Y, Kim YC, Han YB, Park Y, Pariza MW, Ntambi JM. The trans-10,cis-12 isomer of conjugated linoleic acid downregulates stearoyl-CoA desaturase 1 gene expression in 3T3-L1 adipocytes. J Nutr. 2000;130(8):1920-4. doi. 10.1093/jn/130.8.1920.

8. Dadarwal D, Adams GP, Hyttel P, Brogliatti GM, Caldwell S, Singh J. (2015). Organelle reorganization in bovine oocytes during dominant follicle growth and regression. Reproductive biology and endocrinology: RB\&E13, 124-124. doi: 10.1186/s12958-015-0122-0.

9. De Lesegno CV, Reynaud K, Pechoux C, Thoumire S, Chastant-Maillard S. Ultrastructure of canine oocytes during in vivo maturation. Mol Reprod Dev. 2008;75(1):115-25. doi. 10.1002/mrd.20763.

10. Den Brok MH, Raaijmakers TK, Colladocamps E, Adema GJ. Lipid Droplets as Immune Modulators in Myeloid Cells. Trends Immunol. 2018;39(5):380-92. doi. 10.1016/j.it.2018.01.012.

11. Dobrzyn A, Dobrzyn P, Lee SH, Miyazaki M, Cohen P, Asilmaz E, et al. Stearoyl-CoA desaturase-1 deficiency reduces ceramide synthesis by downregulating serine palmitoyltransferase and increasing beta-oxidation in skeletal muscle. Am J Physiol Endocrinol Metab. 2005;288(3):E599. doi. 10.1152/ajpendo.00439.2004.

12. Dowhan W. The role of phospholipids in cell function. Advances in Lipobiology. 1997;2:79-107. doi. 10.1016/S1874-5245(97)80006-7.

13. Elis S, Desmarchais A, Maillard V, Uzbekova S, Monget P, Dupont J. Cell proliferation and progesterone synthesis depend on lipid metabolism in bovine granulosa cells. Theriogenology. 2015;83(5):840-53. doi. 10.1016/j.theriogenology.2014.11.019.

14. Feldstein AE, Werneburg NW, Canbay A, Guicciardi ME, Bronk SF, Rydzewski R, et al. Free fatty acids promote hepatic lipotoxicity by stimulating TNF-alpha expression via a lysosomal pathway. Hepatology. 2004;40(1):185-94. doi. 10.1002/hep.20283.

15. Gallardo-Montejano VI, Saxena G, Kusminski CM, Yang C, Mcafee JL, Hahner L, et al. Nuclear Perilipin 5 integrates lipid droplet lipolysis with PGC-1 a/SIRT1-dependent transcriptional regulation of mitochondrial function. Nat Commun. 2016;7:12723. doi. 10.1038/ncomms12723.

16. Gao G, Chen FJ, Zhou L, Su L, Xu D, Xu L, et al. (2017). Control of lipid droplet fusion and growth by CIDE family proteins. Biochimica et Biophysica Acta (BBA) - Molecular and Cell Biology of Lipids 1197. doi: 10.1016/j.bbalip.2017.06.009.

17. Gao S, Gan X, He H, Hu S, Deng Y, Chen X, et al. (2019). Dynamic characteristics of lipid metabolism in cultured granulosa cells from geese follicles at different developmental stages. Biosci Rep 39(12). https://doi: 10.1042/BSR20192188. 
18. Gilbert A, Evans A, Perry M, Davidson M. A method for separating the granulosa cells, the basal lamina and the theca of the preovulatory ovarian follicle of the domestic fowl (Gallus domesticus). J Reprod Fertil. 1977;50(1):179-81. doi. 10.1530/jrf.0.0500179.

19. Goldberg IJ, Karen R, Abumrad NA, Bickel PE, Sarah C, Fisher EA, et al. Deciphering the Role of Lipid Droplets in Cardiovascular Disease: A Report From the 2017 National Heart, Lung, and Blood Institute Workshop. Circulation An Official Journal of the American Heart Association. 2018;138(3):305-15. doi. 10.1161/CIRCULATIONAHA.118.033704.

20. GURAYA, and S., S.J.J.R.F. A histochemical study of pre-ovulatory and post-ovulatory follicles in the rabbit ovary. J Reprod Fertil. 1968;15(3):381-7. doi. 10.1530/jrf.0.0150381.

21. Han L, Pang K, Li XI, Loor JJ, Yang Gy, Gao T. Lipidomic profiling analysis of the phospholipid molecules in SCAP-induced lipid droplet formation in bovine mammary epithelial cells. Prostaglandins Other Lipid Mediat. 2020;149:106420. doi. 10.1016/j.prostaglandins.2020.106420.

22. Hu W, Qiao JJE. (2011). Expression and regulation of adipocyte fatty acid binding protein in granulosa cells and its relation with clinical characteristics of polycystic ovary syndrome. Endocrine 40(2), 196-202. https://doi: 10.1007/s12020-011-9495-9.

23. Iwai T, Kume S, Chin-Kanasaki M, Kuwagata S, Araki H, Takeda N, et al. (2016). Stearoyl-CoA Desaturase-1 Protects Cells against Lipotoxicity-Mediated Apoptosis in Proximal Tubular Cells. Int J Mol Sci 17(11). doi: 10.3390/ijms17111868.

24. Khor VK, Shen W, Kraemer FB. Lipid droplet metabolism. Current Opinion in Clinical Nutrition Metabolic Care. 2013;16(6):632-7. doi. 10.1097/MC0.0b013e3283651106.

25. Lange M, Ni Z, Criscuolo A, Fedorova MJC. (2018). Liquid Chromatography Techniques in Lipidomics Research. Chromatographia 82(2). https://doi: 10.1007/s10337-018-3656-4.

26. Listenberger L, Townsend E, Rickertsen C, Hains A, Brown E, Inwards EG, et al. (2018). Decreasing Phosphatidylcholine on the Surface of the Lipid Droplet Correlates with Altered Protein Binding and Steatosis. Cells 7(12). https://doi: 10.3390/cells7120230.

27. Liu H. (2017). Special Topic: Metabolomics and Lipidomics. Journal of Analysis Testing 1(3), 185-6. https://doi: 10.1007/s41664-017-0037-1.

28. Liu Z, Xun, HJ.A.B.BS. Lipid metabolism in Drosophila: development and disease. Acta Biochim Biophys Sin. 2013;(1):44-50. doi. 10.1093/abbs/gms105.

29. Lydic TA, Goo YHJC, Medicine T. (2018). Lipidomics unveils the complexity of the lipidome in metabolic diseases. Clinical Translational Medicine 7(1), 4. https://doi: 10.1186/s40169-018-0182-9.

30. Mao X, Cai T, Olyarchuk JG, Wei L. Automated genome annotation and pathway identification using the KEGG Orthology (KO) as a controlled vocabulary. Bioinformatics. 2005;21(19):3787. doi. $10.2307 / 1592215$.

31. Matthew T, Flowers., James MN. Role of stearoyl-coenzyme A desaturase in regulating lipid metabolism. Current Opinion in Lipidology https://. 2008. doi:10.1097/MOL.0b013e3282f9b54d.

32. Mcevoy TG, Coull GD, Broadbent PJ, Hutchinson JSM, Speake BKJR. Fatty acid composition of lipids in immature cattle, pig and sheep oocytes with intact zona pellucida. Journal of Reproduction 
Fertility. 2000;118(1):163-70. doi. 10.1530/jrf.0.1180163.

33. Mike H, Reese M, Joel, and Journal GJE. The assembly of lipid droplets and their roles in challenged cells. Embo Journal. 2019;2(9):e101816. doi. 38 ). : 10.15252/embj.2019101816.

34. Olzmann JA, Carvalho P. (2019). Dynamics and functions of lipid droplets. Nature Reviews Molecular Cell Biology 20(3), 137-155. https://doi: 0.1038/s41580-018-0085-z.

35. Onal G, Kutlu O, Gozuacik D, Dokmeci Emre S. (2017). Lipid Droplets in Health and Disease. Lipids Health Dis 16(1), 128. https://doi: 10.1186/s12944-017-0521-7.

36. Ozcan U, Yilmaz E, Ozcan L, Furuhashi M, Vaillancourt E, Smith RO, et al. Chemical chaperones reduce ER stress and restore glucose homeostasis in a mouse model of type 2 diabetes. Science. 2006;313(5790):1137-40. doi. 10.1126/science.1128294.

37. Pathmaperuma AN, Maña P, Cheung SN, Kugathas K, Josiah A, Koina ME, et al. Fatty acids alter glycerolipid metabolism and induce lipid droplet formation, syncytialisation and cytokine production in human trophoblasts with minimal glucose effect or interaction. Placenta. 2010;31(3):230-9. doi. 10.1016/j.placenta.2009.12.013.

38. Paulini F, Silva RC, Rôlo JLJdP, Lucci CM. Ultrastructural changes in oocytes during folliculogenesis in domestic mammals. Journal of ovarian research. 2014;7:102-2. doi. 10.1186/s13048-014-01026.

39. Sangha GK, Guraya SS. Biochemical changes in lipids during follicular growth and corpora lutea formation and regression in rat ovary. Indian J Exp Biol. 1989;27(11):998-1000.

40. Santos AL, Preta G. (2018). Lipids in the cell: organisation regulates function. Cell Mol Life Sci 75(11), 1909-27. https://doi: 10.1007/s00018-018-2765-4.

41. Sharma RK, Vats R, Sawhney AK. Changes in follicular lipids during follicular growth in the goat (Capra hircus) ovary. Small Ruminant Research. 1996;20(2):177-80. doi. 10.1016/09214488(95)00793-8.

42. Silva RC, Báo SN, Jivago JLPR, Lucci CM. Ultrastructural characterization of porcine oocytes and adjacent follicular cells during follicle development: Lipid component evolution. Theriogenology. 2011;76(9):1647-57. doi. 10.1016/j.theriogenology.2011.06.029.

43. Storck EM, Ozbalci C, Eggert, US.J.A.R.o.B. Lipid Cell Biology: A Focus on Lipids in Cell Division. Annu Rev Biochem. 2018;87(1):839-69. doi. 10.1146/annurev-biochem-062917-012448.

44. Tang QQ. Lipid metabolism and diseases. Science Bulletin. 2016;61(19):1471. doi. 10.1007/s11434016-1174-z.

45. Walker AK, Jacobs RL, Watts JL, Rottiers V, Jiang K, Finnegan DM, et al. A conserved SREBP1/phosphatidylcholine feedback circuit regulates lipogenesis in metazoans. Cell. 2011;147(4):84052. doi. 10.1016/j.cell.2011.09.045.

46. Welte MA, Gould AP. (2017). Lipid droplet functions beyond energy storage. Biochimica et Biophysica Acta (BBA) - Molecular and Cell Biology of Lipids 1862(10, Part B), 1260-1272. doi: 10.1016/j.bbalip.2017.07.006. 
47. Wen R, Gan X, Hu S, Gao S, Deng Y, Qiu J, et al. Evidence for the existence of de novo lipogenesis in goose granulosa cells. Poultry science. 2019;98(2):1023-30. doi. 10.3382/ps/pey400.

48. Xun S, Juan L, Xiaoju Z, Joel G, R?Dk?R SV, F?Rgeman NJ, et al. Regulation of lipid droplet size and phospholipid composition by stearoyl-CoA desaturase. J Lipid Res. 2013;54(9):2504-14. doi. 10.1194/jlr.M039669.

49. Yenuganti VR, Viergutz T, Vanselow J. Oleic acid induces specific alterations in the morphology, gene expression and steroid hormone production of cultured bovine granulosa cells. Gen Comp Endocrinol. 2016;232:134-44. doi. 10.1016/j.ygcen.2016.04.020.

50. Young MD, Wakefield MJ, Smyth GK, Oshlack A. Gene ontology analysis for RNA-seq: accounting for selection bias. Genome Biol. 2010;11(2):1-12. doi. 10.1186/gb-2010-11-2-r14.

51. Yuan X, Hu S, Li L, Liu H, He H, Wang J. (2020). Metabolomic Analysis of SCD during Goose Follicular Development: Implications for Lipid Metabolism. Genes (Basel) 11(9). doi: 10.3390/genes 11091001 .

\section{Tables}


Table 1 Statistical analysis of differential lipids for four cross-comparisons.

\begin{tabular}{|c|c|c|c|c|c|c|}
\hline Molecular species & Vip & Fold change & $p$-vaule & Vip & Fold change & $p$-vaule \\
\hline & \multicolumn{3}{|c|}{ Comparison I: LN vs. LS comparison } & \multicolumn{3}{|c|}{ Comparison II: LG vs. LS comparison } \\
\hline $\mathrm{FFA}(22: 2)$ & 1.64 & 1.81 & 0.094 & 2.38 & 1.54 & 0.141 \\
\hline $\mathrm{PC}(18: 0 / 14: 1)$ & 1.56 & 0.83 & 0.219 & 1.21 & 0.83 & 0.467 \\
\hline $\mathrm{PC}(16: 0 / 20: 3)$ & 2.10 & 0.68 & 0.038 & 2.83 & 0.71 & 0.054 \\
\hline $\mathrm{PC}(18: 1 / 18: 4)$ & 1.97 & 0.61 & 0.032 & 2.07 & 0.77 & 0.168 \\
\hline $\mathrm{PC}(20: 5 / 18: 0)$ & 1.25 & 1.28 & 0.251 & 1.81 & 1.29 & 0.224 \\
\hline $\mathrm{PC}(18: 2 / 20: 3)$ & 1.46 & 0.64 & 0.259 & 1.12 & 1.26 & 0.626 \\
\hline $\mathrm{CE}(20: 4)$ & 1.42 & 1.52 & 0.198 & 1.71 & 1.32 & 0.321 \\
\hline DG(18:2/22:4/0:0) & 1.38 & 0.79 & 0.341 & 1.37 & 1.27 & 0.440 \\
\hline LPC(18:3/0:0) & 1.21 & 1.52 & 0.334 & 1.04 & 1.23 & 0.503 \\
\hline $\mathrm{PC}(18: 2 / 20: 4)$ & 1.38 & 0.67 & 0.163 & 1.46 & 0.75 & 0.354 \\
\hline $\mathrm{PC}(\mathrm{O}-18: 2 / 20: 2)$ & 1.28 & 1.53 & 0.228 & 1.16 & 1.24 & 0.480 \\
\hline $\mathrm{PE}(22: 2 / 12: 0)$ & 1.37 & 0.55 & 0.158 & 2.01 & 0.43 & 0.171 \\
\hline $\operatorname{PE}(22: 2 / 14: 0)$ & 1.70 & 1.55 & 0.145 & 1.36 & 1.21 & 0.386 \\
\hline $\operatorname{PE}(20: 1 / 20: 5)$ & 1.52 & 1.87 & 0.081 & 2.33 & 1.55 & 0.131 \\
\hline PS (20:4/20:0) & 1.04 & 1.23 & 0.359 & 2.23 & 1.22 & 0.199 \\
\hline PS(20:5/18:0) & 1.36 & 1.40 & 0.239 & 1.33 & 1.23 & 0.389 \\
\hline $\mathrm{SM}(\mathrm{d} 18: 0 / 18: 0)$ & 1.13 & 0.71 & 0.306 & 1.14 & 0.71 & 0.444 \\
\hline $\mathrm{SM}(\mathrm{d} 18: 0 / 18: 1)$ & 1.52 & 1.42 & 0.156 & 2.20 & 0.78 & 0.195 \\
\hline TG(14:0/18:0/18:0) & 1.53 & 1.41 & 0.154 & 1.52 & 0.83 & 0.315 \\
\hline TG(14:0/22:0/22:2) & 1.94 & 2.12 & 0.035 & 2.13 & 1.48 & 0.195 \\
\hline TG(14:0/22:1/22:2) & 1.20 & 1.57 & 0.251 & 2.13 & 1.48 & 0.233 \\
\hline TG(12:0/18:1/18:3) & 1.73 & 1.42 & 0.107 & 1.95 & 0.79 & 0.196 \\
\hline TG(14:0/18:2/18:3) & 2.09 & 1.60 & 0.012 & 1.58 & 0.76 & 0.369 \\
\hline TG(16:0/16:1/18:4) & 1.87 & 1.51 & 0.048 & 1.61 & 0.82 & 0.288 \\
\hline TG(14:0/18:4/20:1) & 1.38 & 1.51 & 0.195 & 2.09 & 0.64 & 0.179 \\
\hline \multirow[t]{2}{*}{ TG(14:0/18:3/22:3) } & 1.06 & 1.80 & 0.404 & 1.08 & 1.56 & 0.488 \\
\hline & \multicolumn{3}{|c|}{ Comparison III: LC vs. LT comparison } & \multicolumn{3}{|c|}{ Comparison IV: LC vs. LF comparison } \\
\hline 15-oxoETE & 1.49 & 1.41 & 0.465 & 1.32 & 1.52 & 0.376 \\
\hline $\operatorname{FFA}(4: 0)$ & 2.58 & 0.63 & 0.048 & 2.20 & 0.71 & 0.707 \\
\hline $\operatorname{FFA}(22: 1)$ & 1.61 & 1.38 & 0.389 & 1.05 & 1.22 & 0.510 \\
\hline $\operatorname{FFA}(20: 2)$ & 1.62 & 1.50 & 0.560 & 1.38 & 1.66 & 0.458 \\
\hline $\operatorname{FFA}(22: 2)$ & 2.44 & 1.65 & 0.089 & 2.01 & 1.66 & 0.077 \\
\hline $\operatorname{FFA}(22: 3)$ & 1.81 & 1.26 & 0.331 & 1.97 & 1.44 & 0.105 \\
\hline $\operatorname{FFA}(24: 6)$ & 2.06 & 2.41 & 0.154 & 1.75 & 2.51 & 0.074 \\
\hline LPG(16:0/0:0) & 1.37 & 0.66 & 0.459 & 1.91 & 1.73 & 0.091 \\
\hline $\mathrm{PC}(16: 0 / 20: 3)$ & 1.78 & 0.63 & 0.300 & 1.34 & 0.63 & 0.331 \\
\hline $\mathrm{PC}(18: 1 / 18: 4)$ & 2.59 & 0.66 & 0.092 & 1.48 & 0.76 & 0.304 \\
\hline $\mathrm{PC}(18: 2 / 20: 3)$ & 2.22 & 1.63 & 0.155 & 2.48 & 1.97 & 0.016 \\
\hline $\operatorname{PC}(20: 1 / 20: 4)$ & 1.37 & 1.22 & 0.646 & 1.19 & 1.27 & 0.583 \\
\hline $\operatorname{PE}(18: 2 / 16: 0)$ & 1.71 & 1.20 & 0.375 & 1.79 & 1.35 & 0.183 \\
\hline $\operatorname{PE}(20: 4 / 14: 0)$ & 1.73 & 1.49 & 0.341 & 2.21 & 1.65 & 0.019 \\
\hline PG(18:0/18:1) & 1.90 & 0.72 & 0.230 & 1.80 & 0.63 & 0.141 \\
\hline $\mathrm{PI}(20: 3 / 18: 0)$ & 1.84 & 0.64 & 0.336 & 1.96 & 0.65 & 0.133 \\
\hline $\mathrm{PI}(20: 4 / 16: 0)$ & 1.77 & 0.67 & 0.212 & 1.36 & 0.74 & 0.482 \\
\hline $\mathrm{PI}(18: 1 / 20: 4)$ & 1.56 & 0.77 & 0.336 & 1.83 & 0.65 & 0.205 \\
\hline $\mathrm{CE}(18: 2)$ & 1.91 & 1.36 & 0.214 & 2.11 & 1.40 & 0.079 \\
\hline CE(20:4) & 2.68 & 1.48 & 0.037 & 1.40 & 1.27 & 0.310 \\
\hline Cer(d18:1/22:0) & 1.87 & 1.25 & 0.251 & 1.81 & 1.33 & 0.184 \\
\hline DG(16:0/18:0/0:0) & 2.70 & 0.75 & 0.076 & 1.66 & 0.82 & 0.181 \\
\hline DG(14:0/18:2/0:0) & 1.42 & 1.37 & 0.356 & 2.08 & 1.51 & 0.068 \\
\hline DG(20:0/18:2/0:0) & 1.47 & 1.32 & 0.319 & 1.77 & 1.55 & 0.102 \\
\hline LPC(18:3/0:0) & 1.75 & 1.62 & 0.276 & 1.05 & 1.20 & 0.578 \\
\hline $\operatorname{LPE}(0: 0 / 22: 4)$ & 2.32 & 1.20 & 0.081 & 2.18 & 1.25 & 0.045 \\
\hline $\mathrm{PC}(18: 0 / 22: 0)$ & 1.82 & 1.21 & 0.338 & 2.12 & 1.31 & 0.063 \\
\hline $\operatorname{PC}(20: 5 / 12: 0)$ & 1.82 & 0.80 & 0.236 & 1.48 & 0.82 & 0.279 \\
\hline $\operatorname{PE}(22: 2 / 12: 0)$ & 2.47 & 1.37 & 0.127 & 2.12 & 1.45 & 0.079 \\
\hline PS(20:4/20:0) & 1.12 & 0.79 & 0.417 & 1.19 & 1.24 & 0.391 \\
\hline TG(14:0/16:0/22:0) & 1.33 & 1.31 & 0.701 & 1.14 & 1.37 & 0.643 \\
\hline TG(16:0/20:0/22:0) & 1.57 & 1.67 & 0.332 & 1.26 & 1.48 & 0.465 \\
\hline TG(14:0/22:0/22:2) & 1.78 & 0.77 & 0.273 & 1.16 & 0.80 & 0.429 \\
\hline TG(14:0/18:1/20:3) & 2.33 & 1.65 & 0.082 & 1.26 & 1.42 & 0.364 \\
\hline
\end{tabular}

Note

$p$-vaule from Student's t test; VIP = variable importance in the projection; 
$\mathrm{FFA}=$ free fat acid; $\mathrm{CE}=$ cholesteryl ester; $\mathrm{DG}=$ diacylglycerol; $\mathrm{TG}=$ triglyceride; $\mathrm{PC}=\mathrm{phosphatidylcholine;}$ $\mathrm{PE}=$ glycerophosphoethanolamine; $\mathrm{PS}=$ glycerophosphoserine; $\mathrm{PG}=$ glycerophosphoglycerol; $\mathrm{PI}=$ glycerophosphoinositol; $\mathrm{LPC}=$ lysophosphatidylcholine; LPG= lysophosphatidylglycerol; Cer=ceramide; SM= sphingomyelin.

Table 2. Statistic of the mapping of sequence to the reference genome goose

\begin{tabular}{|c|c|c|c|c|c|c|}
\hline Terms & NC & OG & OS & SC & ST & SF \\
\hline Clean reads (percentage ${ }^{\mathrm{a}}$ ) & $51566021(96.10 \%)$ & $53774948(96.57 \%)$ & $55702230(96.37 \%)$ & $57694766(96.62 \%)$ & $51644681(96.54 \%)$ & $56187441(96.41 \%)$ \\
\hline Reads mapped (percentage ${ }^{\mathrm{b}}$ ) & $38589491(74.91 \%)$ & $39550763(73.60 \%)$ & 40979329(73.37) & $43575018(75.62 \%)$ & $38710384(75.00 \%)$ & $42250540(75.19 \%)$ \\
\hline Uniquely mapped & $37687479(73.16 \%)$ & $38664803(71.96 \%)$ & 40043890(71.69) & $42597240(73.92 \%)$ & $37884321(73.40 \%)$ & $41295405(73.49 \%)$ \\
\hline
\end{tabular}

\section{Figures}

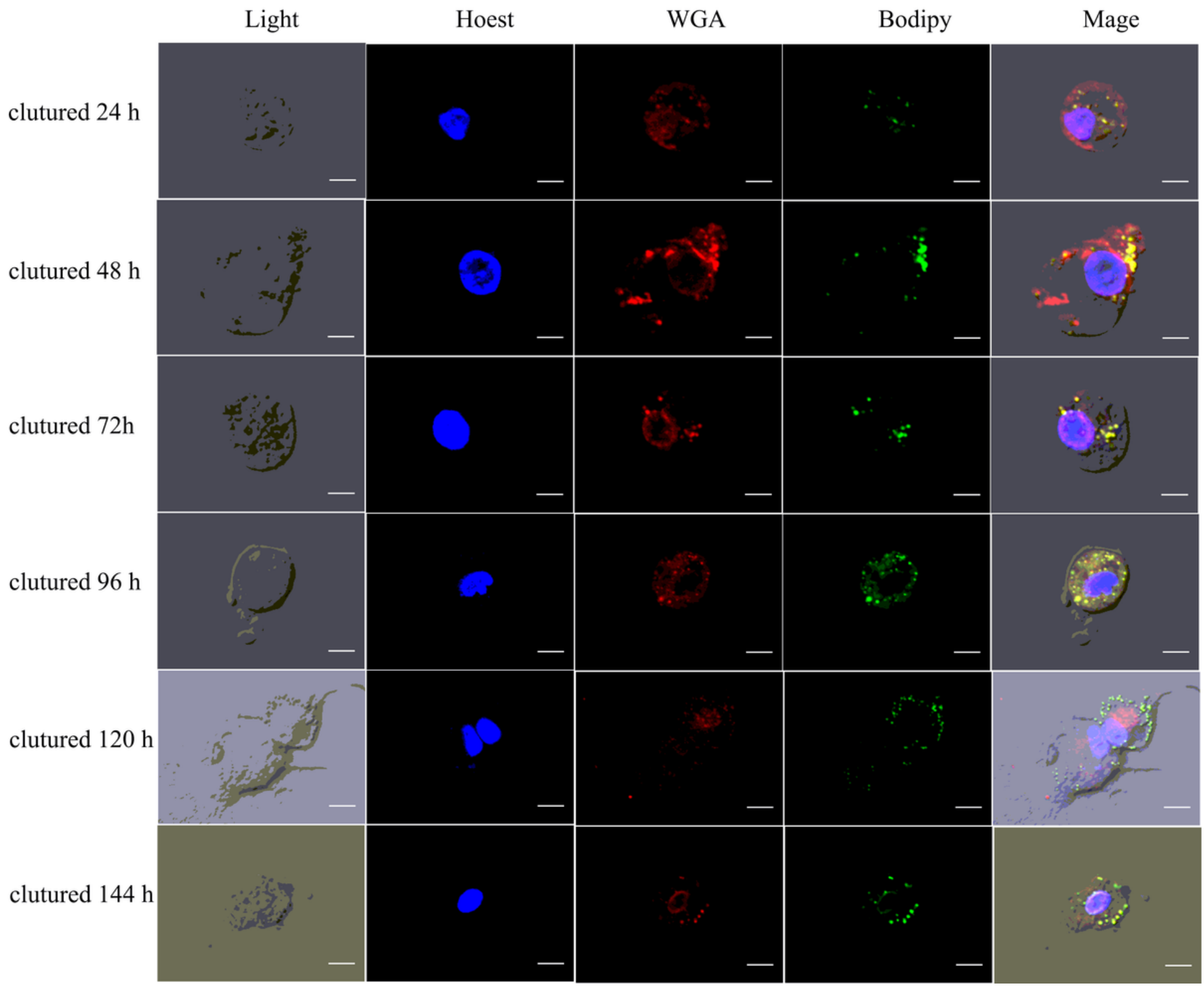




\section{Figure 1}

Distribution of lipid droplets (LDs) and their colocalization in live goose granulosa cells. LDs were stained with the neutral lipid dye Bodipy 500/510 (green), cell membrane were stained with WGA (red), the nuclei were stained with Hoechst 33258 (blue). All images are equal magnification, scale bar indicates $40 \mu \mathrm{m}$.

A

GFP

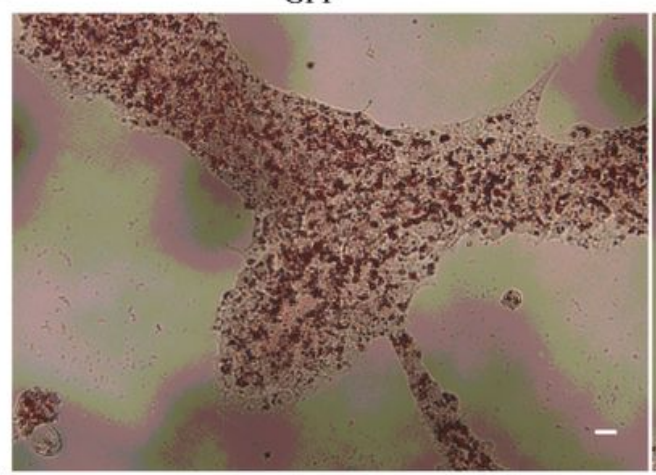

B

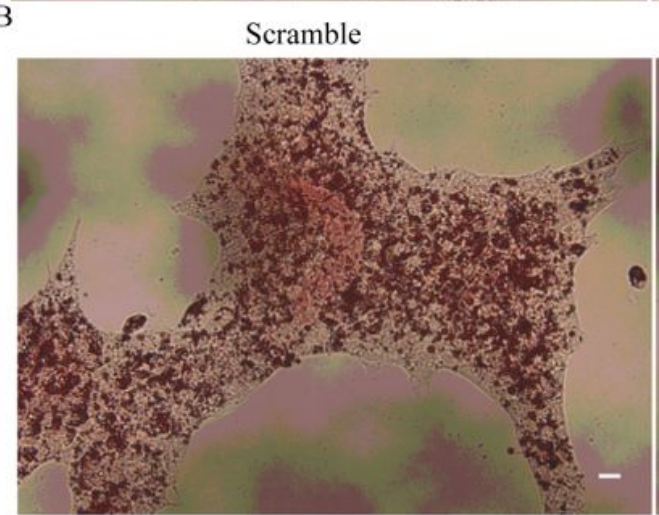

$\mathrm{C}$

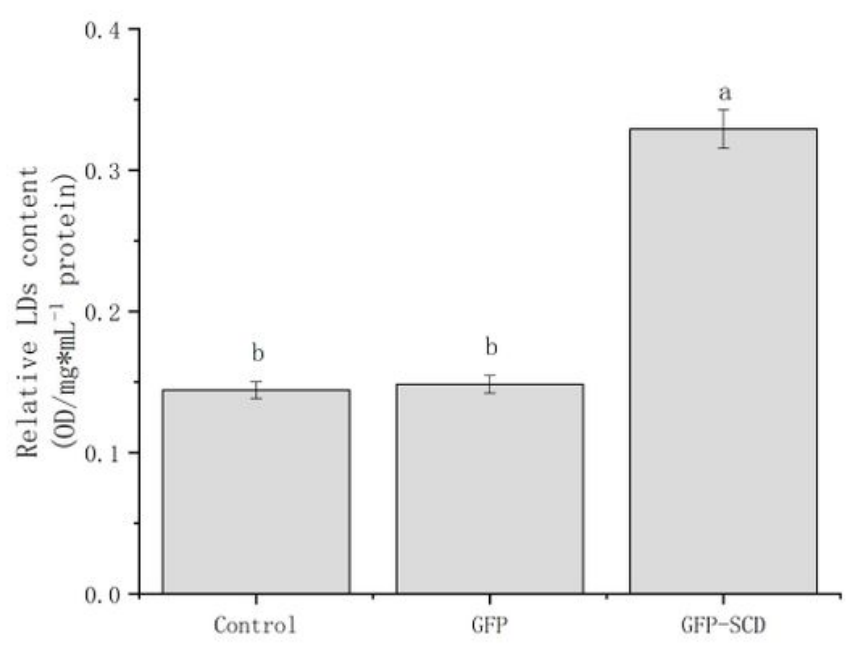

Control

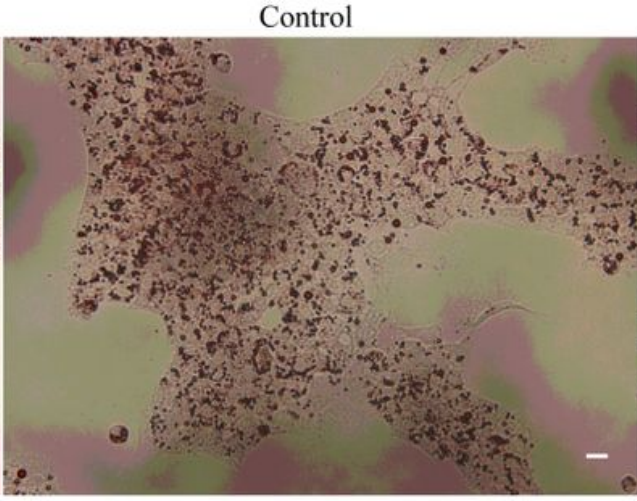

SiRNA-210

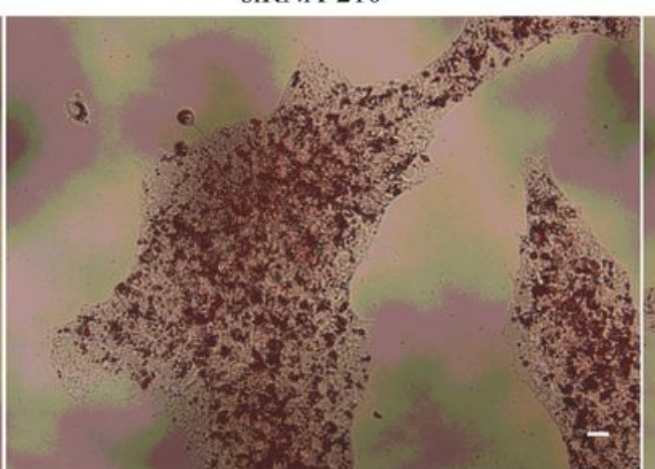

GFP-SCD

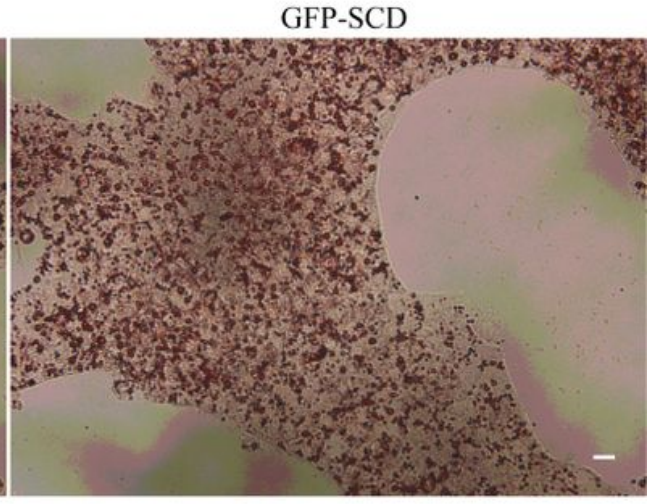

siRNA-470
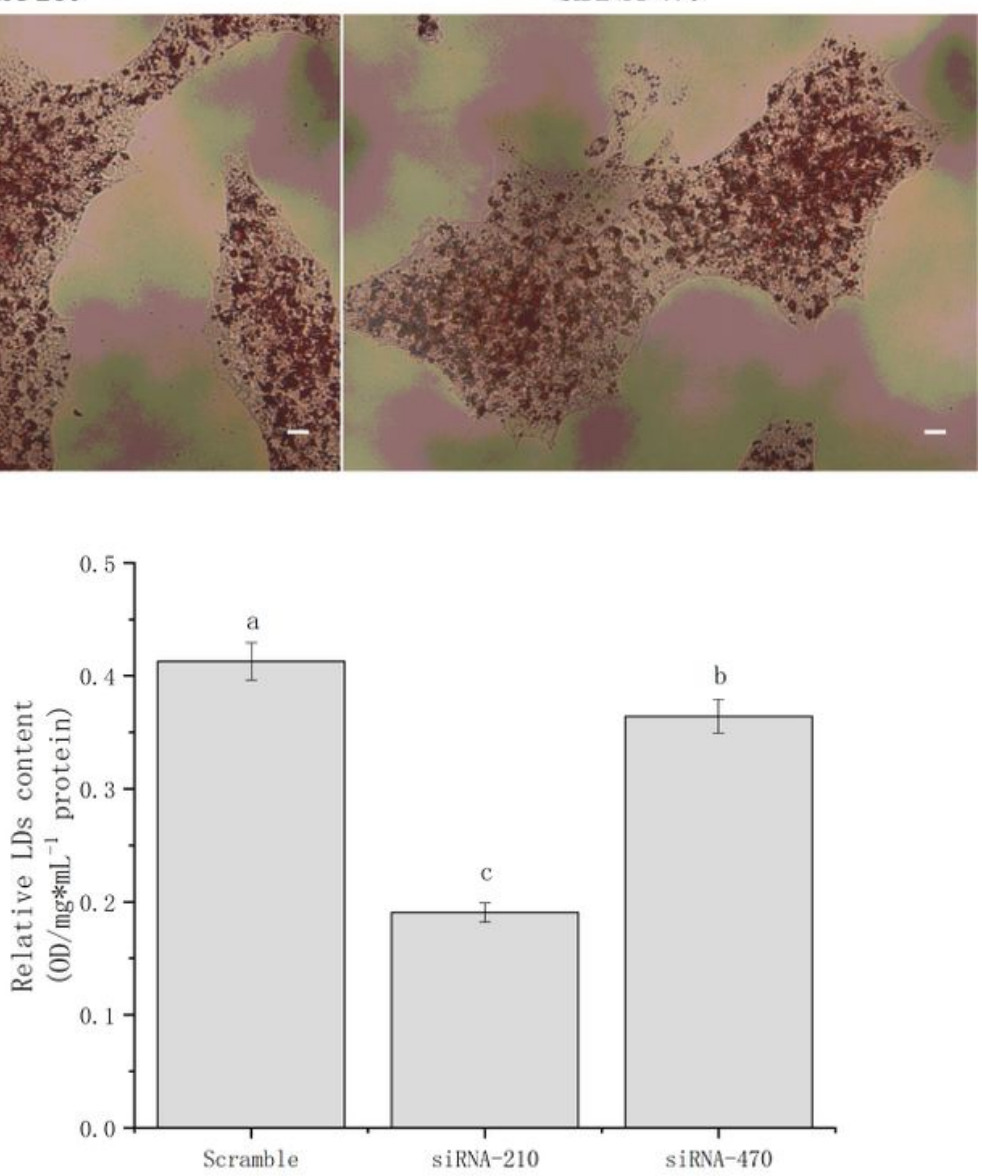

\section{Figure 2}

After culturing, cells were fixed with formaldehyde and stained with Oil red $\mathrm{O}$, scale bar indicates $200 \mu \mathrm{m}$. (A) The cells were after SCD expression. (B) The cells were after SCD knockdown. (C) TG amount in each cell sample was shown as normalized with amount of cellular protein. The results are shown as 
mean \pm S.D. from six experiments, the data were analyzed by ANOVA and Tukey's test. The lowercase letter indicates significant differences between each group $(P<0.05)$.

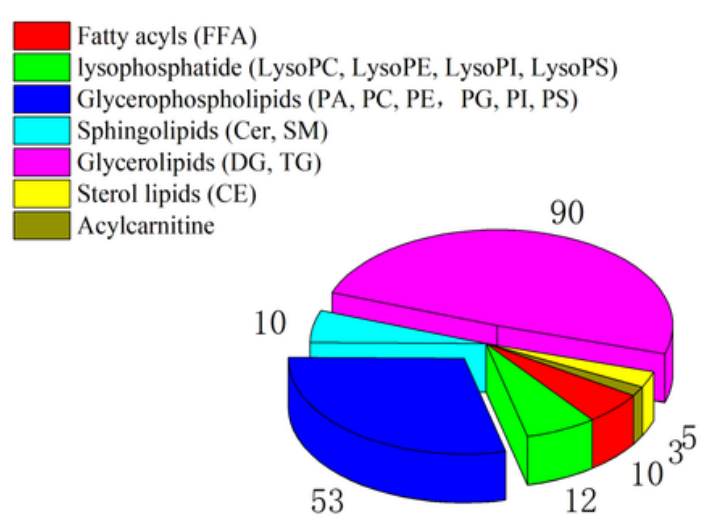

LN vs. LS

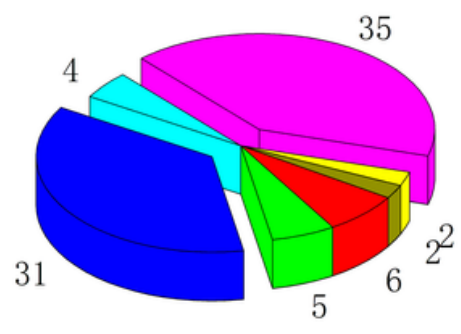

LG vs. LS

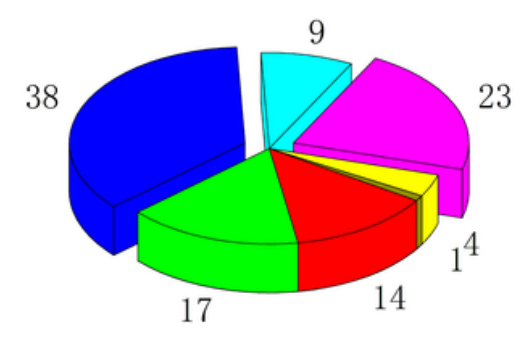

LC vs. LT

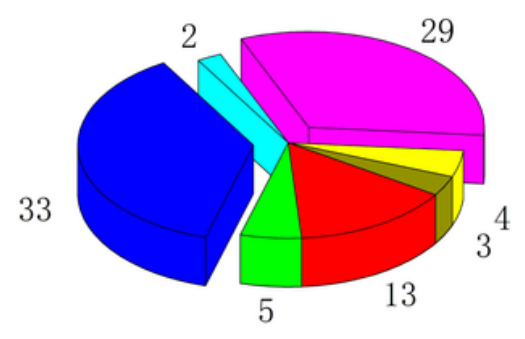

LC vs. LF

\section{Figure 3}

Lipidomics studies was performed in a cellular model of SCD function of goose GCs, pie chart shows number of significantly disturbed lipids of each group. 
A

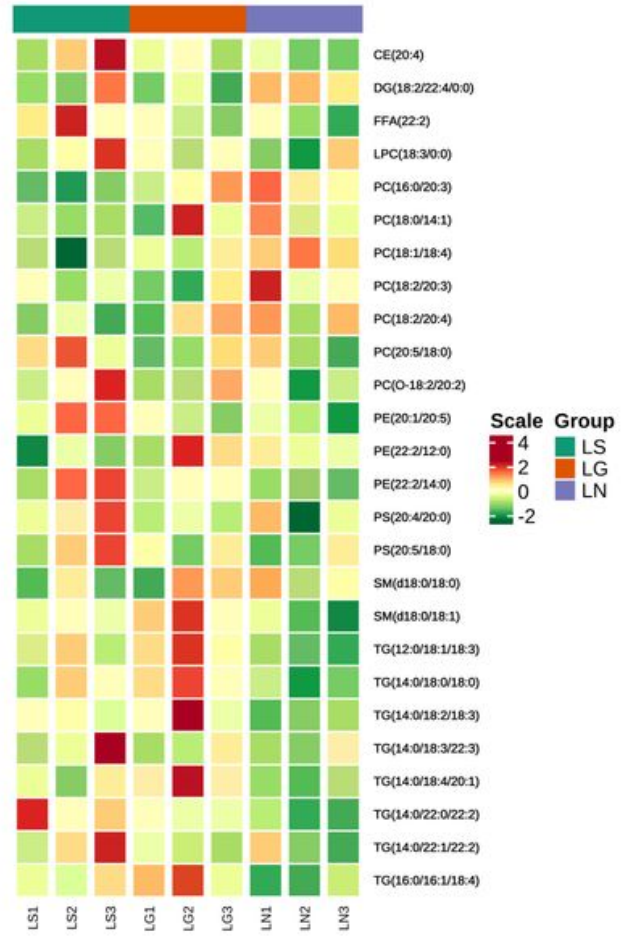

B

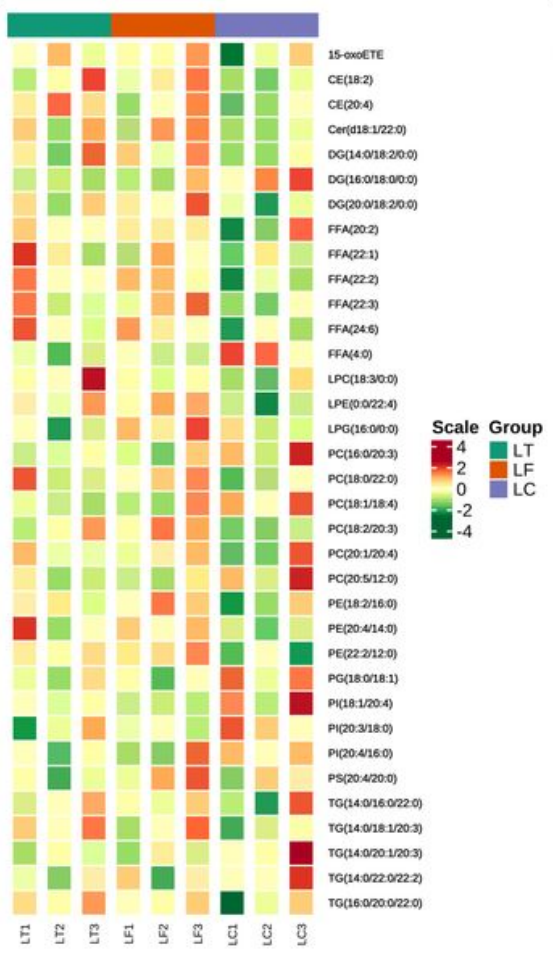

C

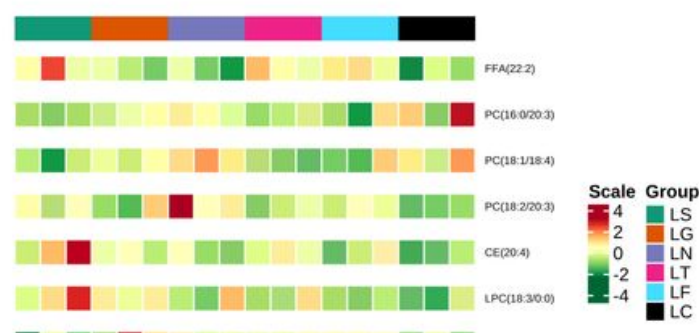

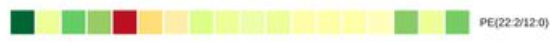
PS(20:420.0) те(14.0230252x

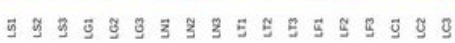

\section{Figure 4}

Heat map from the hierarchical clustering of differential overlapping lipid in the (A) SCD-overexpression group; (B) SCD-knockdown group; (C) SCD-overexpression and SCD-knockdown groups. The scaled expression by row (lipids) is shown as a heat map and is reordered by a hierarchical clustering analysis (Pearson's distance and Ward's method) on both rows and columns. The color scale indicates the relative amounts of metabolites: red, higher levels; green, lower levels. 
A

LN vs. LS

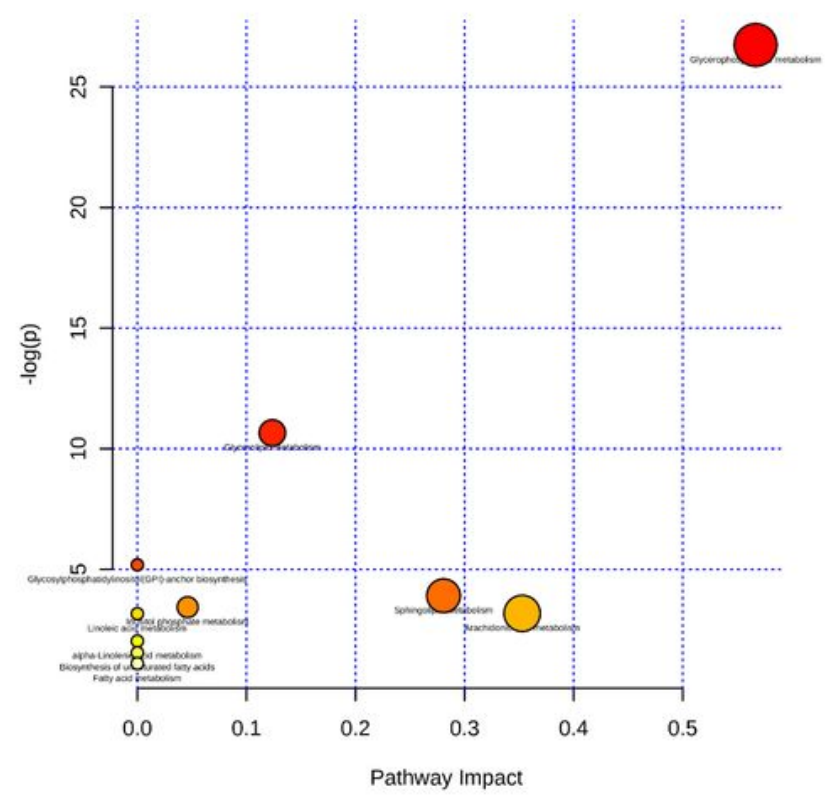

B

LC vs. LT

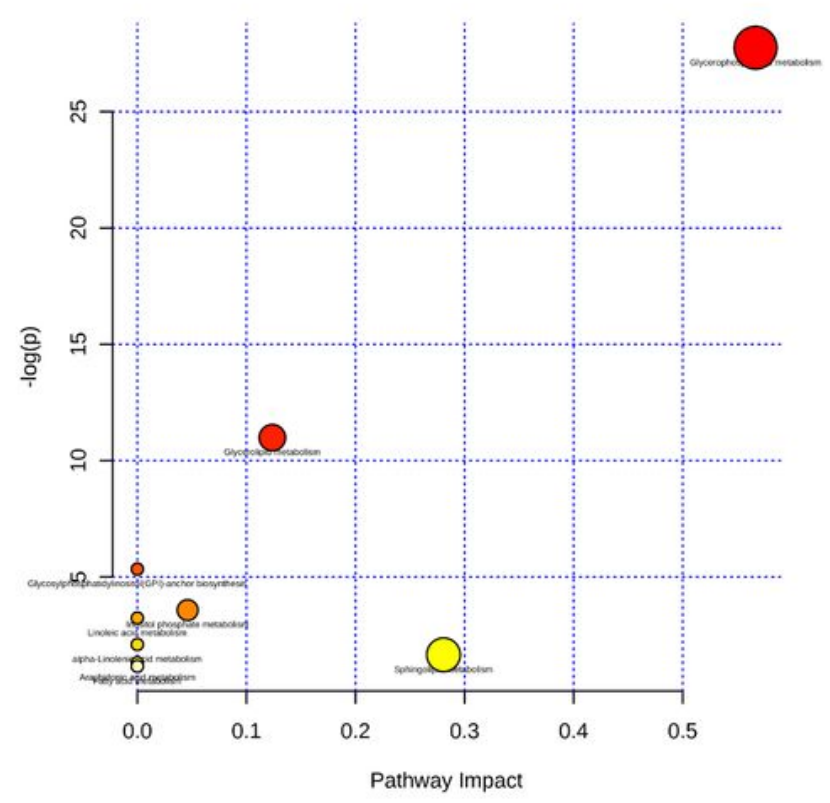

LG vs. LS

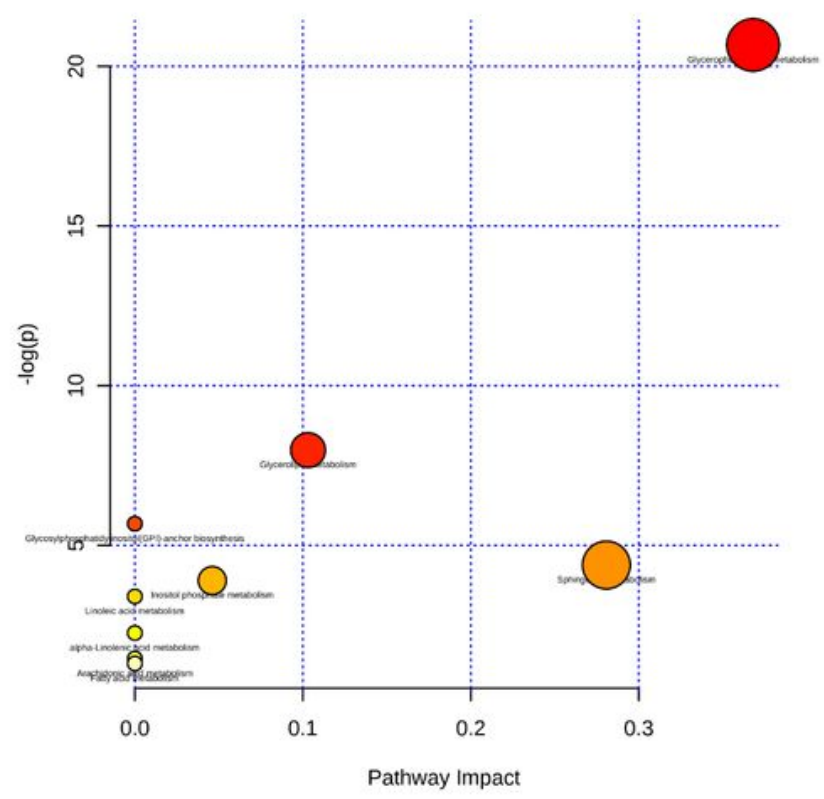

LC vs. LF

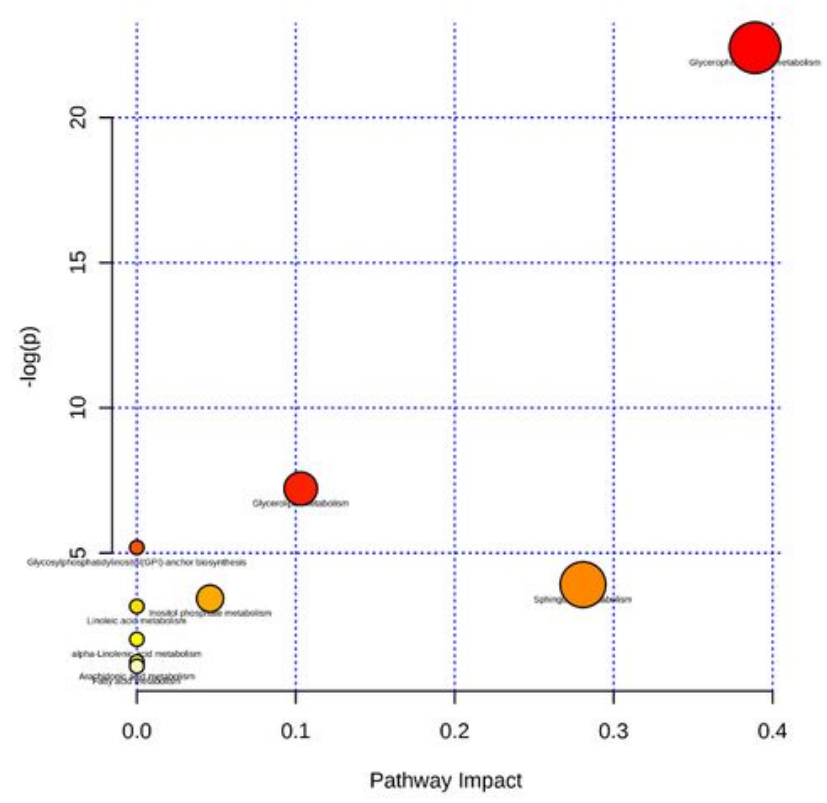

\section{Figure 5}

Disturbed metabolic pathways. Metaboanalyst (http://www.metaboanalyst.ca) generated topology map described the impact of baseline metabolites identified with high VIP values $(\mathrm{VIP}>1)$ on metabolic pathways between (A) Overexpressed group, LN vs. LS comparison and LG vs. LS comparison; (B) Knockdown group, LC vs. LT comparison and LC vs. LF comparison. 
A

A

UDOWN

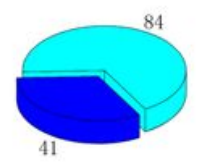

NC vs. OS

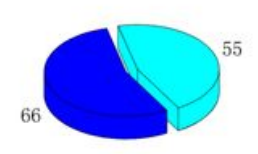

OG vs. OS

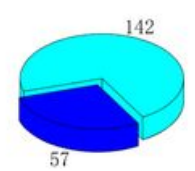

SC vs. ST

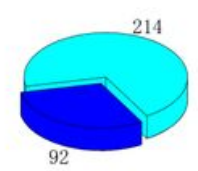

SC vs. SF
B

\section{venn}

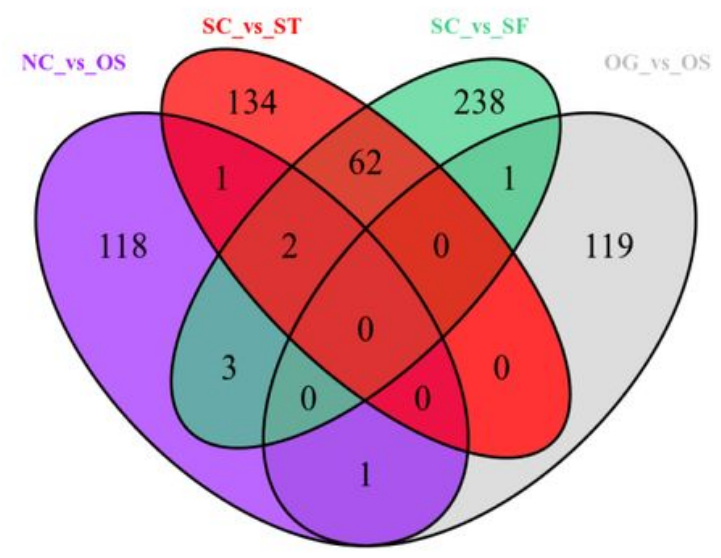

\section{Figure 6}

Transcriptomics studies was performed in a cellular model of SCD function of goose GCs. (A) Pie chart shows number of significantly disturbed DEGs of each group. (B) Venn diagram of overlapping and unique of DEGs altered in each group. 
A

NC vs. OS

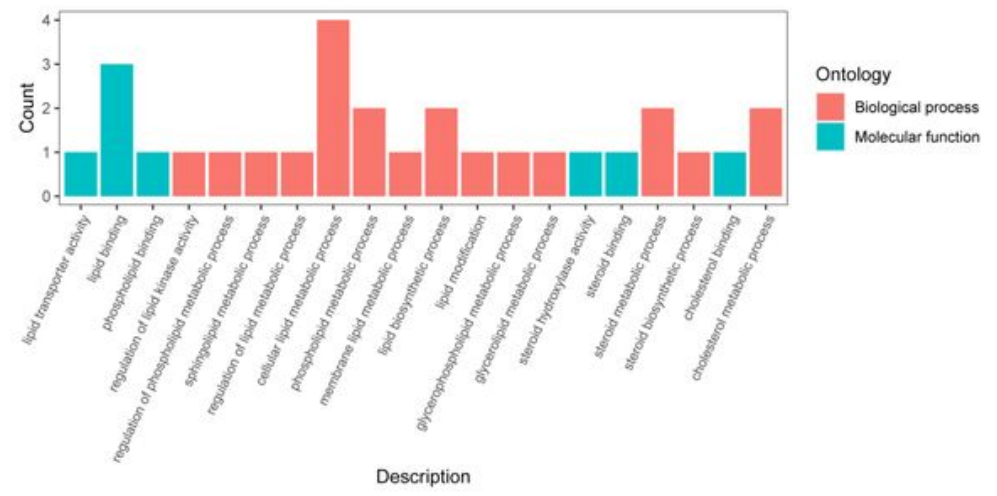

B

OG vs. OS
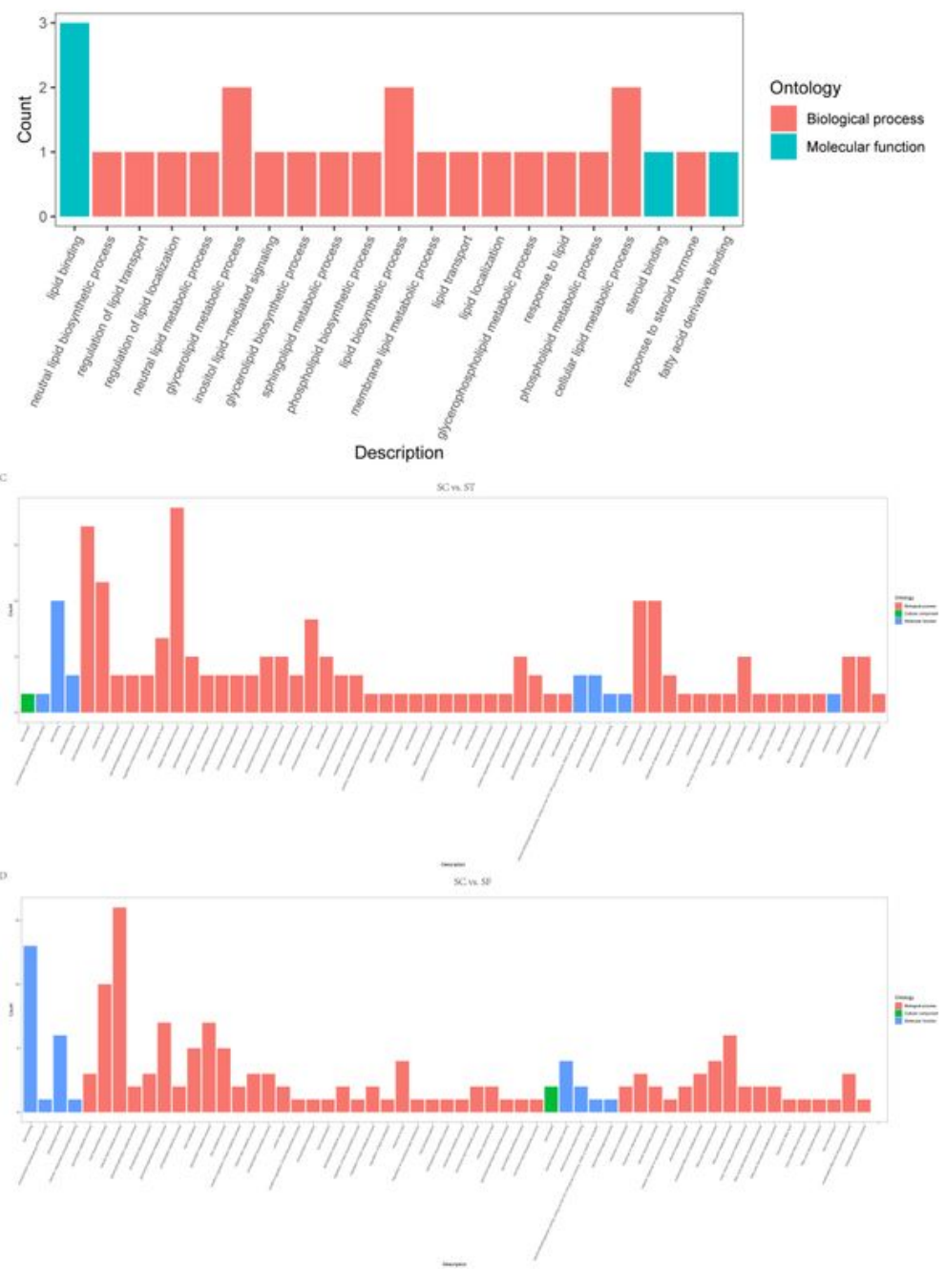

Figure 7

Histogram presentation of the lipid-related Gene Ontology classification of the differentially expressed DEGs. (A) NC vs. OS comparison; (B) OG vs. OS comparison; (C) SC vs. ST comparison; (D) SC vs. SF comparison. The results of GO classifications are provided in Supplementary Table 4. 
A

LN-NC vs. LS-OS

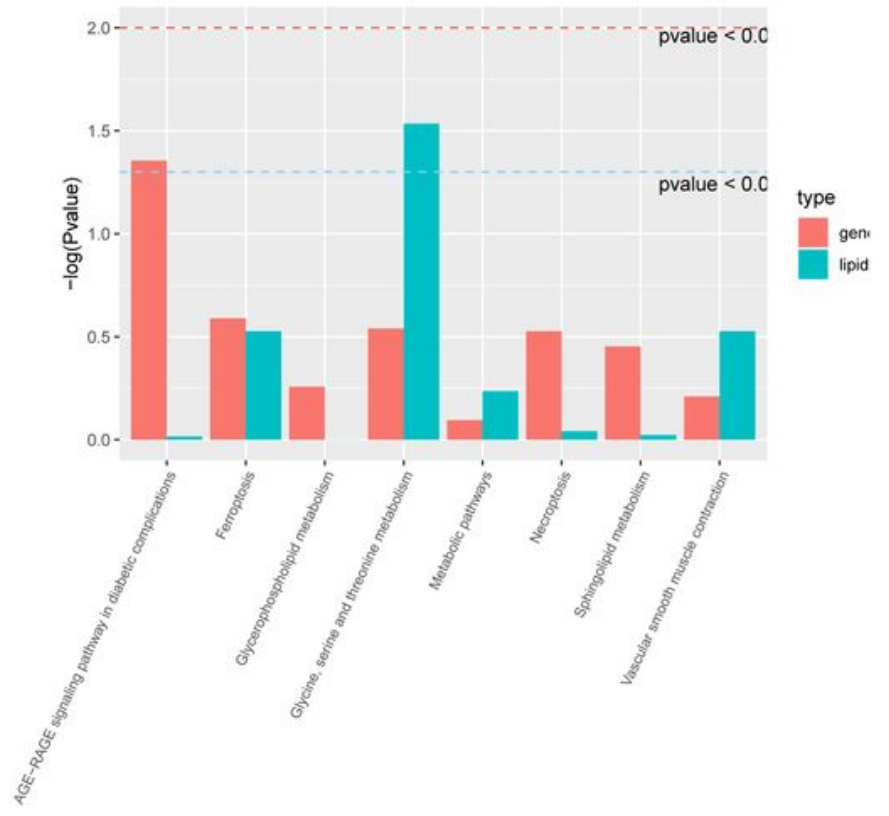

B

LC-SC vs. LT-ST

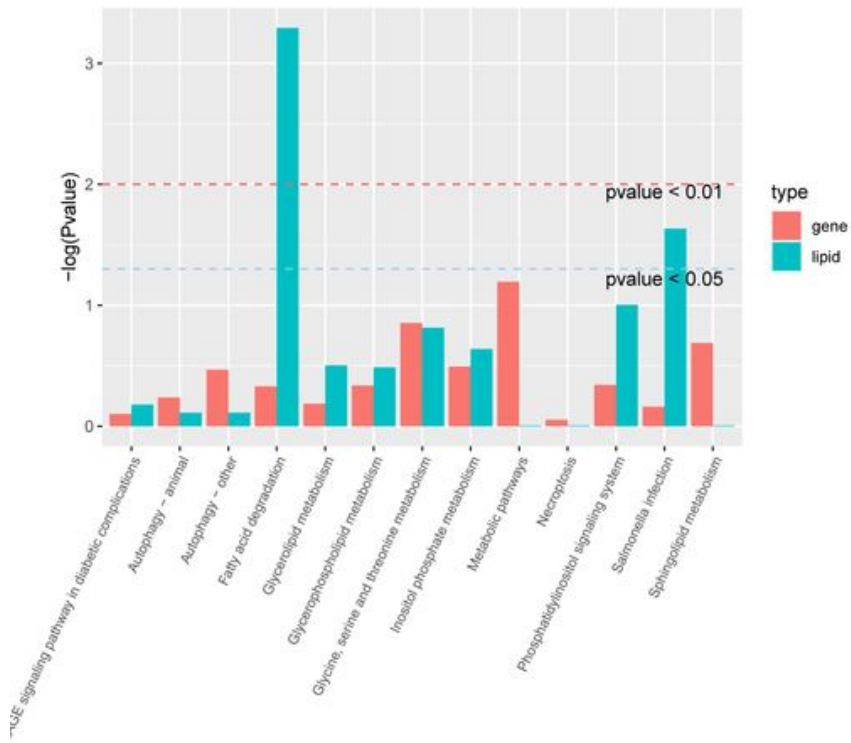

LG-OG vs. LS-OS

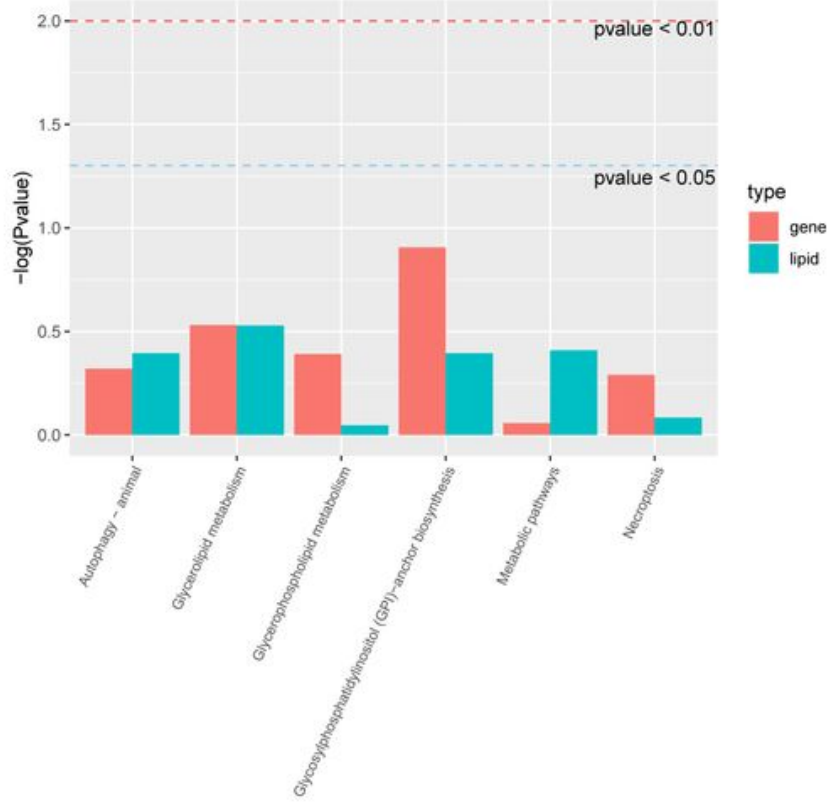

LC-SC vs. LF-SF

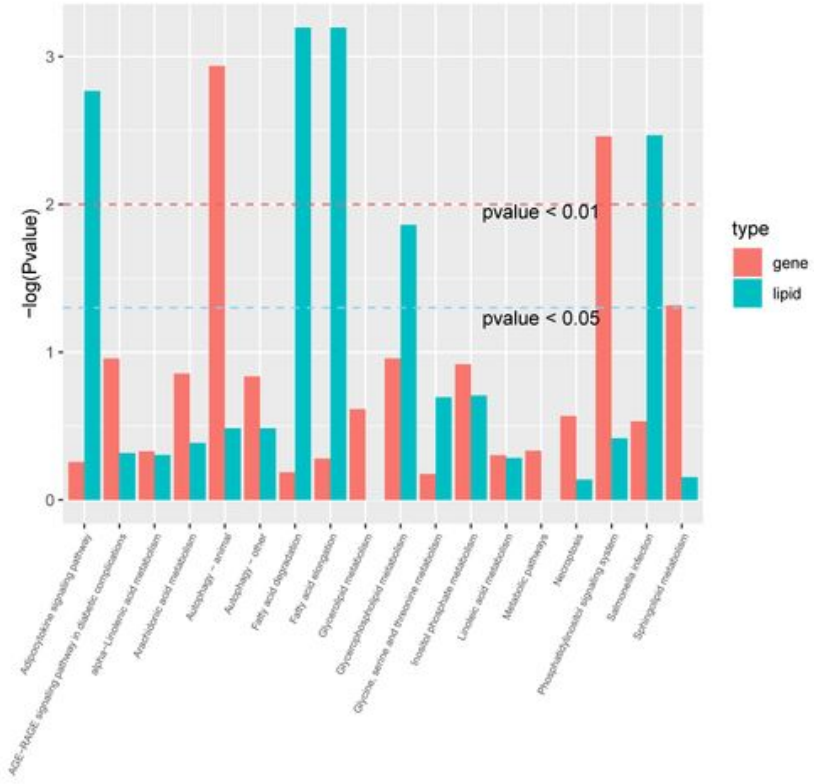

\section{Figure 8}

Two-omics datasets into pathway enrichment analysis. (A) LN-NC vs. LS-OS comparison and LG-OG vs. LS-OS comparison; (B) LC-SC vs. LT-ST comparison and LC-SC vs. LF-SF comparison; 

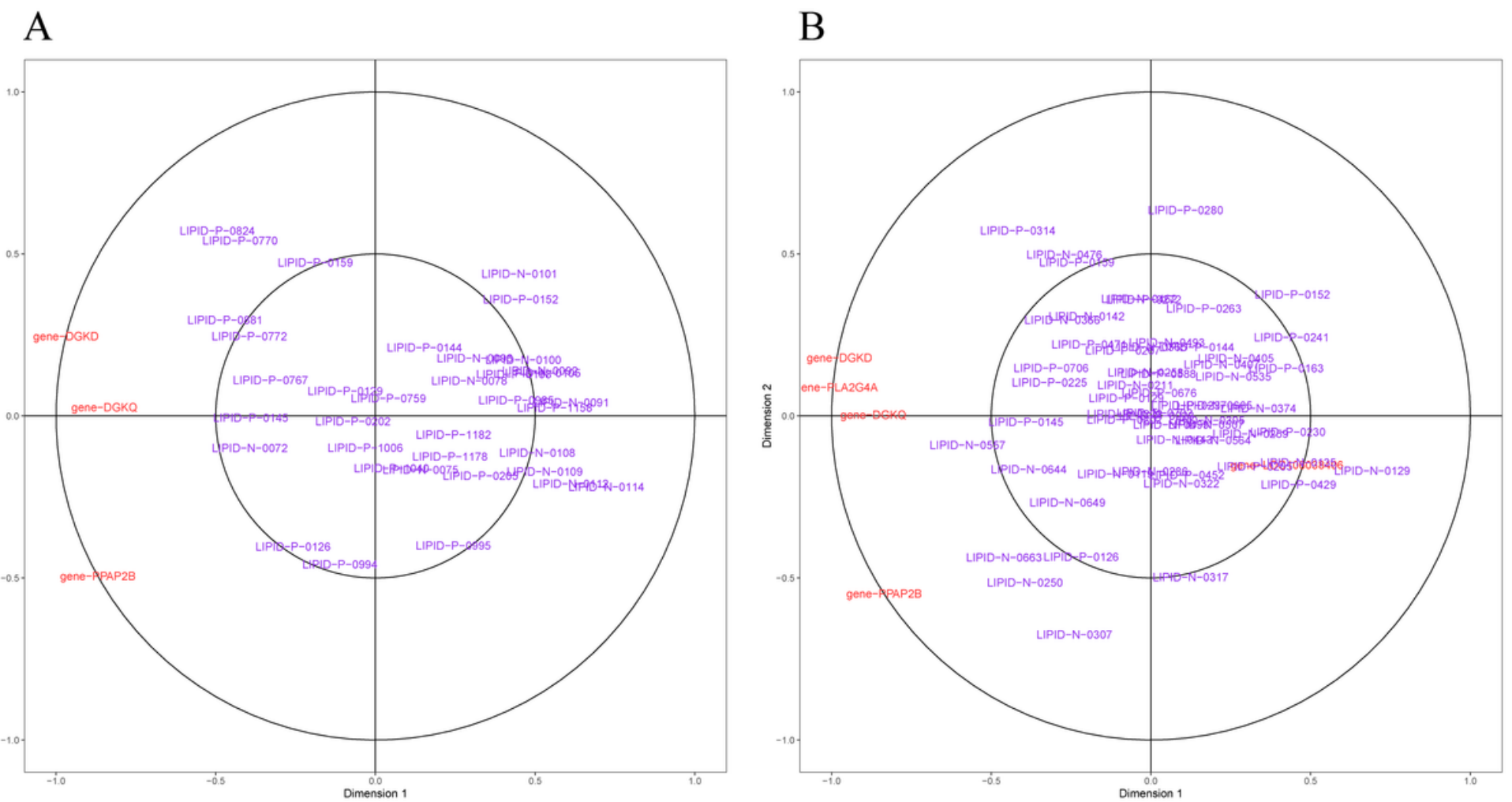

Figure 9

Visualization of the CCA results of lipids and DEGs involved in (A) glycerolipid metabolism and (B) glycerophospholipid metabolism under SCD knockdown.

\section{Supplementary Files}

This is a list of supplementary files associated with this preprint. Click to download.

- SupplementaryFigure1.pdf

- SupplementaryFigure2.tif

- SupplementaryFigure3.pdf

- SupplementaryFigure4A.tif

- SupplementaryFigure4B.tif

- SupplementaryFigure5.tif

- SupplementaryTable1.docx

- SupplementaryTable2.docx

- SupplementaryTable3.docx

- SupplementaryTable4.docx 